Darío Alejandro Rossignolo*

Universidad de Buenos Aires

Buenos Aires, Argentina

Recibido: 8 de junio de 2017

Concepto de evaluación: 24 de junio de 2017

Aprobado: 27 de junio de 2017

Artículo de investigación

(C) 2017 Universidad Católica de Colombia.

Facultad de Ciencias

Económicas y Administrativas.

Todos los derechos reservados

* Doctor en Economía, Magíster en Economía y Licenciado en Economía. Profesor de la Universidad de Buenos Aires, Buenos Aires, Argentina. Dirección de correspondencia: Lavalle 1772 1 ro 2do, Buenos Aires, Argentina. Correo electrónico: darossignolo@ economicas.uba.ar
Finanz. polit. econ., ISSN: 2248-6046, Vol. 9, No. 2, julio-diciembre, 2017, pp. 215-247

http://dx.doi.org/10.14718/revfinanzpolitecon.2017.9.2.2

\section{El esfuerzo fiscal en los países de América Latina y el Caribe}

\section{RESUMEN}

En América Latina, la recaudación fiscal ha experimentado un crecimiento en los últimos quince años; sin embargo, los niveles de desigualdad y pobreza imperantes llevan a preguntarse si los países pueden aumentar aún en mayor medida los ingresos tributarios, en función de poder financiar en forma genuina los niveles de gasto público necesarios para morigerarlos. Mediante la metodología de esfuerzo fiscal, en este artículo se presentan estimaciones que permiten concluir que los países con menor nivel de desarrollo están asociados a una menor capacidad de generación de recursos fiscales, y países con desarrollo medio o alto podrían incrementar su recaudación, pero por decisiones políticas e institucionales han determinado mantener tasas impositivas bajas.

Palabras clave: características institucionales, esfuerzo fiscal, frontera estocástica, recursos naturales.

JEL: H3, H2, H7, D2

\section{Fiscal effort in the countries of Latin America and the Caribbean}

\section{ABSTRACT}

In Latin America, tax revenue has increased in the last fifteen years; nevertheless, the prevailing levels of inequality and poverty lead to the question of whether these countries can increase tax revenues even more, in order to be able to genuinely finance public expenditure levels that are necessary to restrain them. Using fiscal effort as metodology, this article presents estimations that allow concluding that countries with lower levels of development have a lower capacity to generate fiscal resources, while countries with medium or high levels of development could be able to increase their tax collection, but due to political and institutional reasons they have decided to maintain low tax rates.

Keywords: institutional characteristics, fiscal effort, stochastic frontier, natural resources. 


\section{O esforço fiscal nos países da América Latina e do Caribe}

\section{RESUMO}

Na América Latina, a arrecadação fiscal tem experimentado um crescimento nos últimos quinze anos; porém, os níveis de desigualdade e pobreza imperantes nos levam a questionar se os países podem aumentar ainda mais os ingressos tributários, em função de poder financiar de forma genuína os níveis de gasto público necessários para amenizá-los. Mediante a metodologia de esforço fiscal, neste artigo, são apresentadas estimativas que permitem concluir que os países com menor nível de desenvolvimento estão associados a uma menor capacidade de geração de recursos fiscais, e países com desenvolvimento médio ou alto poderiam incrementar a sua arrecadação, mas por decisões políticas e institucionais determinaram manter taxas impositivas baixas.

Palavras-chave: características institucionais, esforço fiscal, fronteira estocástica, recursos naturais. 


\section{INTRODUCCIÓN}

Es un hecho conocido que América Latina es la región más desigual del planeta (Fondo Monetario Internacional [FMI], 2014), realidad que es agravada particularmente por los vaivenes del contexto macroeconómico que determinan cuadros de extrema volatilidad, lo que hace que se verifiquen aumentos significativos en la inequidad y la pobreza. A partir de la acción de los gastos públicos y los impuestos, los diferentes países pueden ayudar a mitigar estos aspectos enfatizando sus efectos redistributivos.

En consecuencia, para los gobiernos de la región es relevante asegurar un nivel de gasto público que pueda servir a los fines de morigerar las fluctuaciones de los ciclos económicos, para lo cual resulta de capital importancia contar con financiamiento genuino a través de los recursos tributarios. Las administraciones tributarias desempeñan un rol central en este proceso, dado que resultan ser el instrumento clave a través del cual deben llevarse a cabo las políticas impositivas.

La presión fiscal total, incluyendo contribuciones sociales, se ha estabilizado en la región en niveles cercanos a superiores al $25 \%$ del producto interno bruto (PIB) a partir de 2005, mayores al promedio de entre 20 y 22 puntos de la década 1990-2001. Los ingresos exclusivamente tributarios -esto es, excluyendo los recursos de la seguridad social- han oscilado en los 20 puntos del PIB, mayores a los 16 y 17 puntos del PIB de la década 1990-2001.

Sin embargo, los referidos incrementos recaudatorios llevan a plantearse la cuestión de si los países de América Latina pueden, en función de sus características económicas, sociales e institucionales, aumentar aún en mayor medida los ingresos tributarios, lo que ampliaría el margen para realizar políticas anticíclicas y redistributivas por parte de los gobiernos de la región. Por ejemplo, los niveles de presión fiscal de la región se encuentran muy por debajo de los países de la Organización para la Cooperación y el Desarrollo Económicos (OCDE). En el periodo 2002-2013, en América Latina y el Caribe la presión fiscal creció a $25 \%$ del PIB, mientras que en los países de la OCDE el indicador se mantuvo prácticamente constante, en valores superiores a los $34,5 \%$ del PIB'

Si bien se han hecho grandes avances en la última década, aún subsisten en la región características particulares que determinan un nivel recaudatorio bajo en relación con parámetros internacionales. Además de la evasión, que continúa teniendo un rol importante, las exenciones, deducciones, alícuotas diferenciales y otras reglamentaciones -el denominado gasto tributario- que permiten la elusión ayudan a reducir la obligación impositiva de los países de América Latina (Jiménez, Gómez Sabaini y Rossignolo, 2012).

En consecuencia, es relevante estimar qué tan factible en términos analíticos resulta el incremento de la recaudación impositiva en los países de América Latina, a través de la metodología de esfuerzo fiscal. Uno de los objetivos de las comparaciones internacionales de esfuerzo fiscal es explicitar si un país se encuentra restringido en sus ingresos fiscales por una baja capacidad tributaria o por un reducido esfuerzo en la explotación de la capacidad fiscal con la que cuenta, y financiar de esa manera el gasto público (Stotsky y WoldeMariam, 1997).

Otro de los propósitos es orientar sobre cuál sería la proporción adecuada de políticas fiscales para afrontar un desequilibrio fiscal; si la recaudación estuviere cercana a su nivel potencial, la reducción del déficit debería llevarse a cabo mediante una disminución del gasto público, dado que no sería posible incrementar los impuestos.

El objetivo de este artículo es efectuar una estimación econométrica teórica del esfuerzo fiscal de los países de América Latina. El procedimiento analítico que se empleará consiste en calcular inicialmente la recaudación fiscal teórica de los países de América Latina, estimándola en relación con el

$1 \quad$ La fuente de información para los datos de ingresos fiscales en América Latina y el Caribe corresponden a la publicación conjunta del Banco Interamericano de Desarrollo y el Centro Interamericano de Administraciones Tributarias (2015): Carga fiscal de América Latina y el Caribe 2015. La información de la OCDE se obtuvo de http://stats.oecd.org/. 
comportamiento de variables exógenas; una vez esta es determinada, la diferencia con la recaudación potencial permitirá apreciar el esfuerzo fiscal.

Para las estimaciones mencionadas se tomarán en cuenta los datos de 23 países de América Latina y el Caribe, en función de la disponibilidad de información, dado que la base de información considerada es la Carga fiscal en América Latina y el Caribe 2015, publicada en forma conjunta por el Banco Interamericano de Desarrollo (BID) y el Centro Interamericano de Administraciones Tributarias (CIAT). Un valor agregado de este artículo, a diferencia de otros trabajos en la materia, es que la estimación tendrá en cuenta la carga fiscal ajustada, que incluye las contribuciones privadas a la seguridad social y otros ingresos fiscales (Barreix et al., 2013), dado que se considera que esta representa de mejor manera a la capacidad de extracción de recursos del sector privado por parte de los hacedores de política.

Si bien existen diferentes metodologías para llevar a cabo estas estimaciones, como los modelos de panel estáticos o dinámicos (Stotsky y WoldeMariam, 1997; Kenney y Winner, 2006; Davoodi y Grigorian, 2007; Gordon y Li, 2009; Gupta, 2007, entre otros), en este trabajo se aplicará el análisis de frontera estocástica (Pessino y Fenocchietto, 2010, 2013), el cual, a similitud de las fronteras de posibilidades de producción, intenta aproximar la estimación de la máxima recaudación alcanzable en función de las variables estructurales de las economías.

El presente documento se estructura de la siguiente manera. En primer lugar, se evalúa el comportamiento de la recaudación fiscal para los países de América Latina, y adicionalmente se compara su evolución y estructura con los países de la OCDE. Posteriormente, se explicitan los antecedentes de este tipo de estudios y sus correspondientes metodologías de estimación, mostrando los resultados alcanzados, con énfasis en los resultados para América Latina.

Más adelante se describe la metodología de análisis para el abordaje de la problemática por analizar, se presentan los resultados de las estimaciones para cada uno de los modelos y se exponen las predicciones del esfuerzo fiscal para cada uno de los modelos y las especificaciones. Ulteriormente, se abordará la contribución de las administraciones tributarias al cumplimiento fiscal de los contribuyentes, procurando establecer algunos lineamientos orientativos referidos al aporte de estas al incremento del esfuerzo fiscal. Por último, se presentan las conclusiones.

En relación con el alcance de este tipo de estudios, debe tenerse en cuenta que una característica importante de estas estimaciones es que, si bien logran establecer apreciaciones sobre el comportamiento de la recaudación impositiva en comparación con la frontera recaudatoria, sus conclusiones deben necesariamente ser tomadas como estimaciones cualitativas sobre la política impositiva de las autoridades tributarias y en ningún caso considerarse como datos determinísticos. De ahí que las conclusiones y las medidas de política sean necesariamente generales y no específicas.

\section{LA EVOLUCIÓN DE LA ESTRUCTURA FISCAL EN AMÉRICA LATINA Y SU COMPARACIÓN CON LOS PAÍSES DE LA OCDE Y LA UNIÓN EUROPEA}

La evolución de la estructura tributaria en los países de América Latina y el Caribe ha presentado dos características distintivas al compararla con la de los países de la OCDE. En primer lugar, los niveles de presión tributaria de la región, pese a haber evidenciado un crecimiento en la última década, resultan aún inferiores en promedio a los de los países avanzados ${ }^{2}$ (gráfico 1). En promedio, para el periodo 1990-2001, la carga tributaria alcanzó $17,2 \%$ del PIB para la región, mientras que en los países de la OCDE registró, en promedio, 34,7 puntos del producto ${ }^{3}$. En América Latina y el Caribe, el periodo 2002-2013 mostró un promedio de 20,2\% del PIB en términos de carga tributaria, valores aún

\footnotetext{
Los países considerados se presentan en la tabla 2.

3 El indicador de presión tributaria de Argentina se construyó a partir de la estimación de la serie de PIB presentada en Cetrángolo, Gómez Sabaini y Morán (2015).
} 
Evolución de la presión tributaria en América Latina y el Caribe y la OCDE (promedios no ponderados)

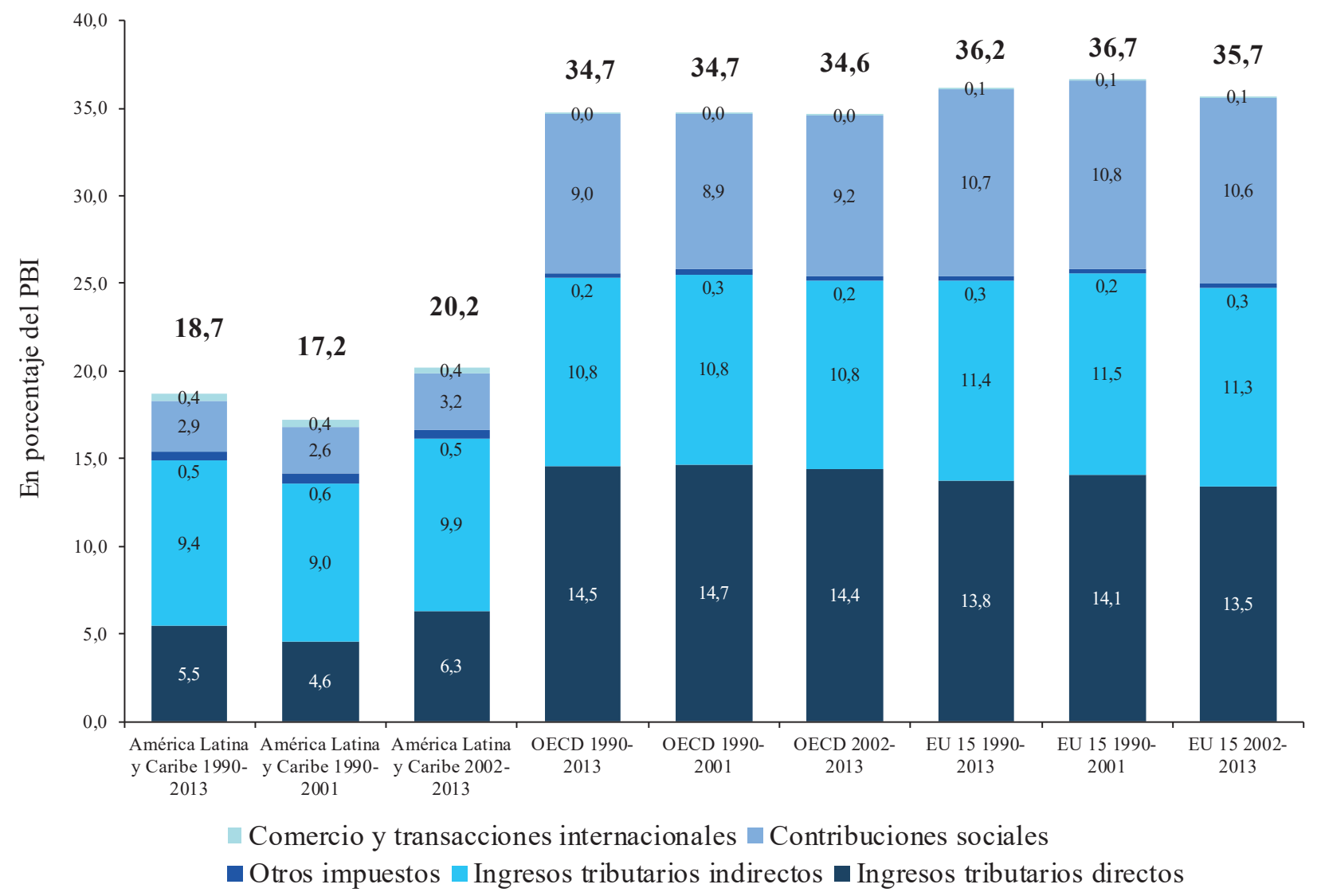

Fuente: cálculos propios basados en CEPALSTAT, OCDE y Database CIAT-BID.

lejanos de los 34,6\% del PIB de la OCDE. Similares conclusiones pueden extraerse a partir de la comparación con los quince países iniciales de la Unión Europea.

En segunda instancia, en la mayoría de los casos se evidencia un crecimiento desigual entre la imposición directa e indirecta (Gómez Sabaini y Rossignolo, 2014). En América Latina y el Caribe, la presión tributaria promedio de los ingresos tributarios indirectos alcanzó 9,9\% del PIB entre 2002-2013, ligeramente superior a los nueve puntos del PIB de 1990-2001. En el caso de la OCDE, la presión tributaria alcanzó los 10,8 puntos del PIB entre 1990-2001 y se mantuvo aproximadamente en el mismo nivel en el periodo posterior. En el periodo 2002-2013, los ingresos tributarios directos representaron para la $\mathrm{OCDE}$, en promedio, un $14,4 \%$ del PIB, similar a los 14,7 puntos del PIB del periodo 1990-2001; para los países de la región, estos ingresos crecieron de $4,6 \%$ del PIB en $1990-$ 2001 a 6,3\% en 2002-2013.

La estructura porcentual de la recaudación tributaria en la región continúa divergiendo de la de los países de la OCDE, aunque se aprecia una mayor similitud en el periodo 2002-2013. Para la OCDE, en promedio, los impuestos directos representaron alrededor del $42 \%$ del total de recursos tributarios para 2002-2013, mientras que los impuestos indirectos significaron el 31,2\%. En América Latina y el Caribe, la mayor proporción de recursos tributarios se relaciona con los impuestos indirectos, los cuales han concentrado un 48,8\% del total en 2002-2013, 
lo cual es ligeramente inferior al periodo anterior. La participación de los impuestos directos es inferior para el periodo 2002-2013 (31,2\%), aunque se ha incrementado en más de cuatro puntos porcentuales respecto de los valores promedio del periodo 1990-2001 (gráfico 2).

Otra diferencia que surge al analizar la composición de la recaudación aparece cuando se visualizan los recursos sobre el comercio exterior. En América Latina, a lo largo de la última década, si bien se ha reducido ligeramente la participación porcentual, la región ha descansado en mayor medida en impuestos a la exportación en lugar de aranceles de importación. Mientras tanto, estos recursos tienen una importancia muy escasa en los países de la OCDE.

La carga tributaria de los países de América Latina y el Caribe se ha estabilizado en valores promedio superiores al $17 \%$ del PIB a partir de mediados de la década del 2000, si se consideran solo los ingresos tributarios. Por su parte, si se consideran los recursos de la seguridad social, los valores promedio rondan el $21 \%$, mientras que la presión fiscal ajustada ha alcanzado cifras promedio superiores al 24\% del PIB (gráfico 3).

En cuanto a la composición porcentual de la presión fiscal ajustada (gráfico 4), se advierte un aumento marcado de la participación de los recursos de la seguridad social tanto públicos como privados, un crecimiento de la imposición directa y una ligera caída en la participación de los impuestos indirectos (en este caso, incluye los recursos del comercio exterior). Sin embargo, al ponderar la presión tributaria de cada país por el valor de su respectivo PIB en relación con el PIB de todos los países analizados, se advierte que la importancia de los impuestos indirectos continúa

Composición de los recursos tributarios en América Latina y la OCDE (promedios no ponderados)

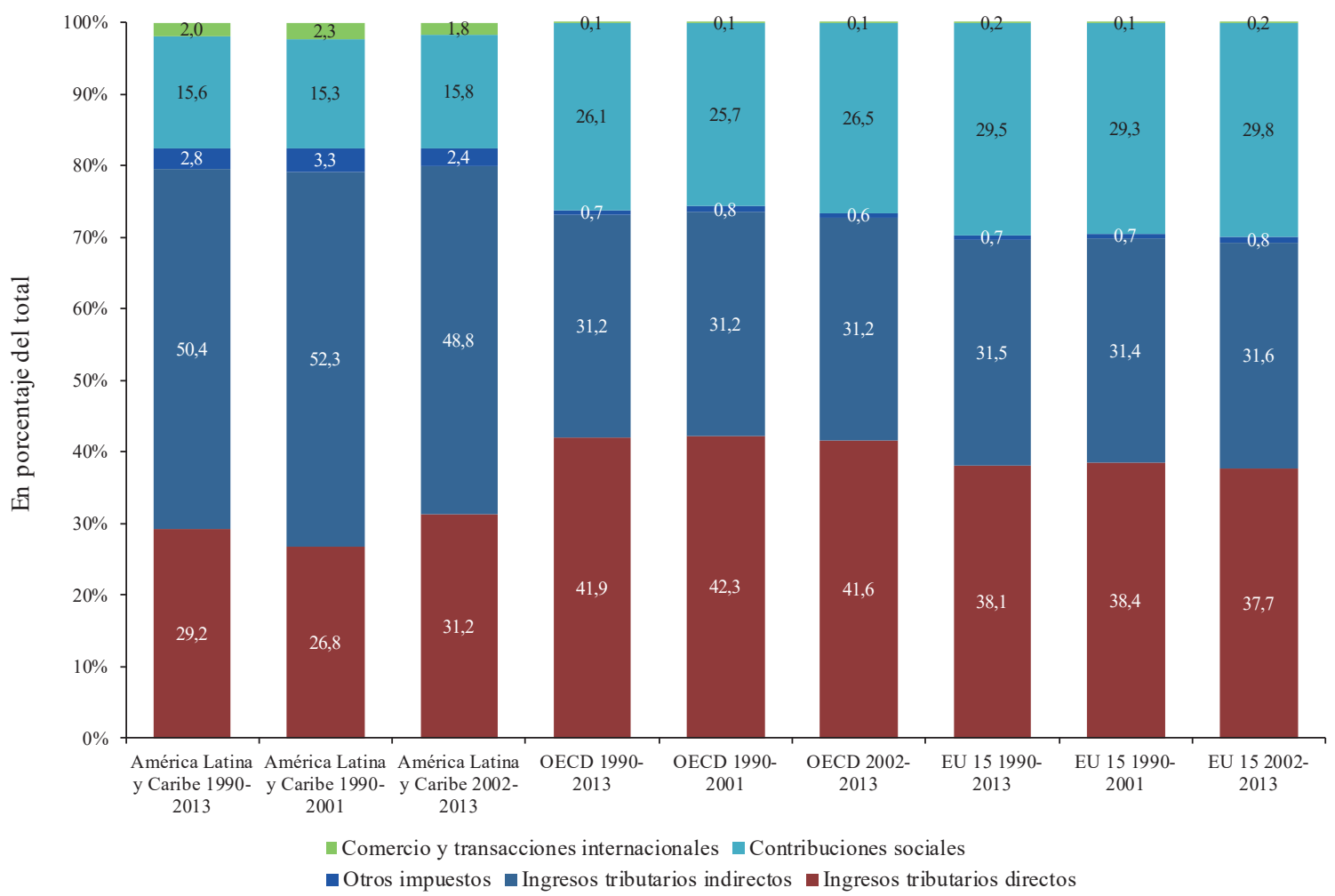

Fuente: cálculos propios basados en CEPALSTAT, OCDE y Database CIAT-IDB. 
Ingresos tributarios, contribuciones a la seguridad social y presión fiscal ajustada (promedios no ponderados en porcentaje del PIB)

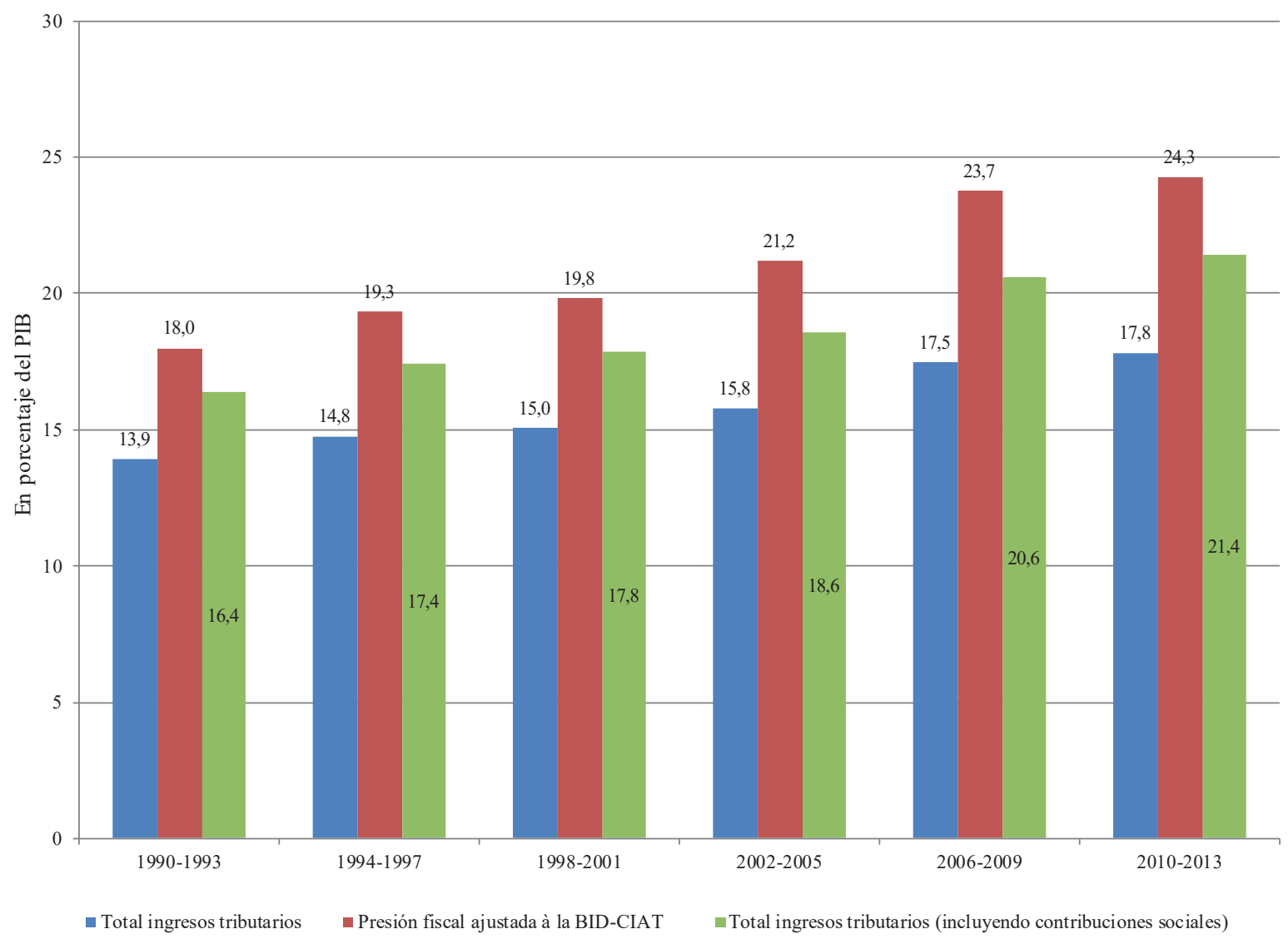

Fuente: Banco Interamericano de Desarrollo (BID) - Centro Internacional de Agricultura Tropical (CIAT).

siendo preponderante respecto a la imposición directa en la región.

Diferentes estudios, como el de Gómez Sabaini y Rossignolo (2014), han hecho notar que el aumento en la carga tributaria en la mayoría de los países de la región se relaciona con tres factores principales, a saber: a) reformas a la administración tributaria; b) introducción de impuestos mínimos, impuestos a las transacciones financieras e impuestos sobre recursos naturales (windfall taxes), y c) un proceso de crecimiento económico impulsado por el auge en los precios de las exportaciones de bienes primarios, el cual generó incrementos en las bases imponibles.
Los ingresos tributarios relacionados con los recursos naturales constituyen una porción relevante de la recaudación total para los países de América Latina. En el gráfico 5 se advierte que, si bien la participación porcentual de estos ha descendido ligeramente luego de haber alcanzado su máximo en 2006, con un share mayor al $27 \%$, su importancia se mantiene en un promedio mayor que en la etapa previa.

En el gráfico 6 se presenta la importancia relativa de los recursos naturales en los ingresos totales, discriminados por país. En Venezuela, Ecuador, México y Bolivia (E. P.), estos recursos han significado proporciones superiores al 30\% del total de ingresos; particularmente en el caso 
Composición de la presión fiscal ajustada promedio en América Latina y el Caribe

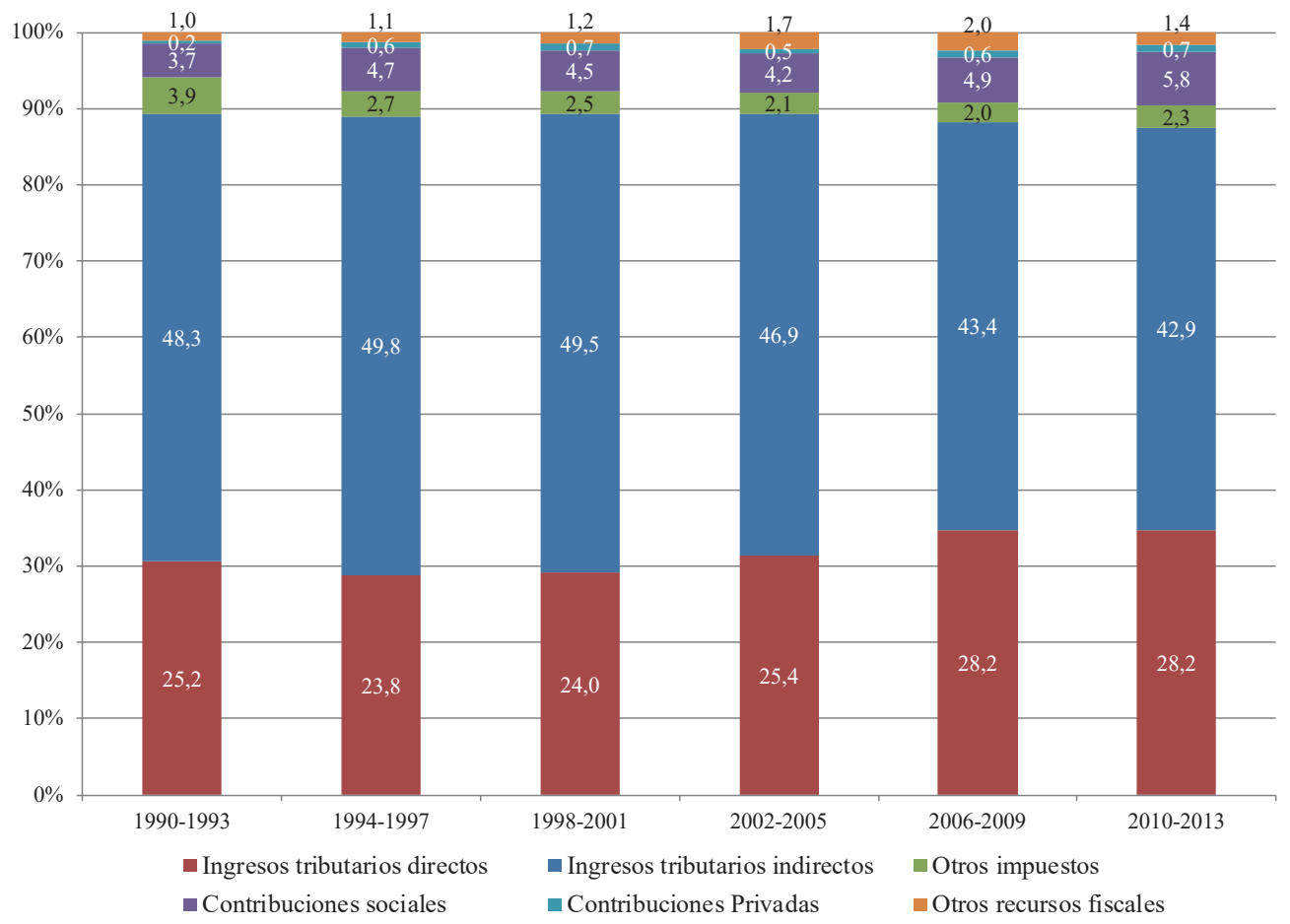

Fuente: Database BID-CIAT y CEPALSTAT.

Gráfico 5.

Estructura porcentual de ingresos fiscales de recursos naturales no renovables, periodo 2000-2012

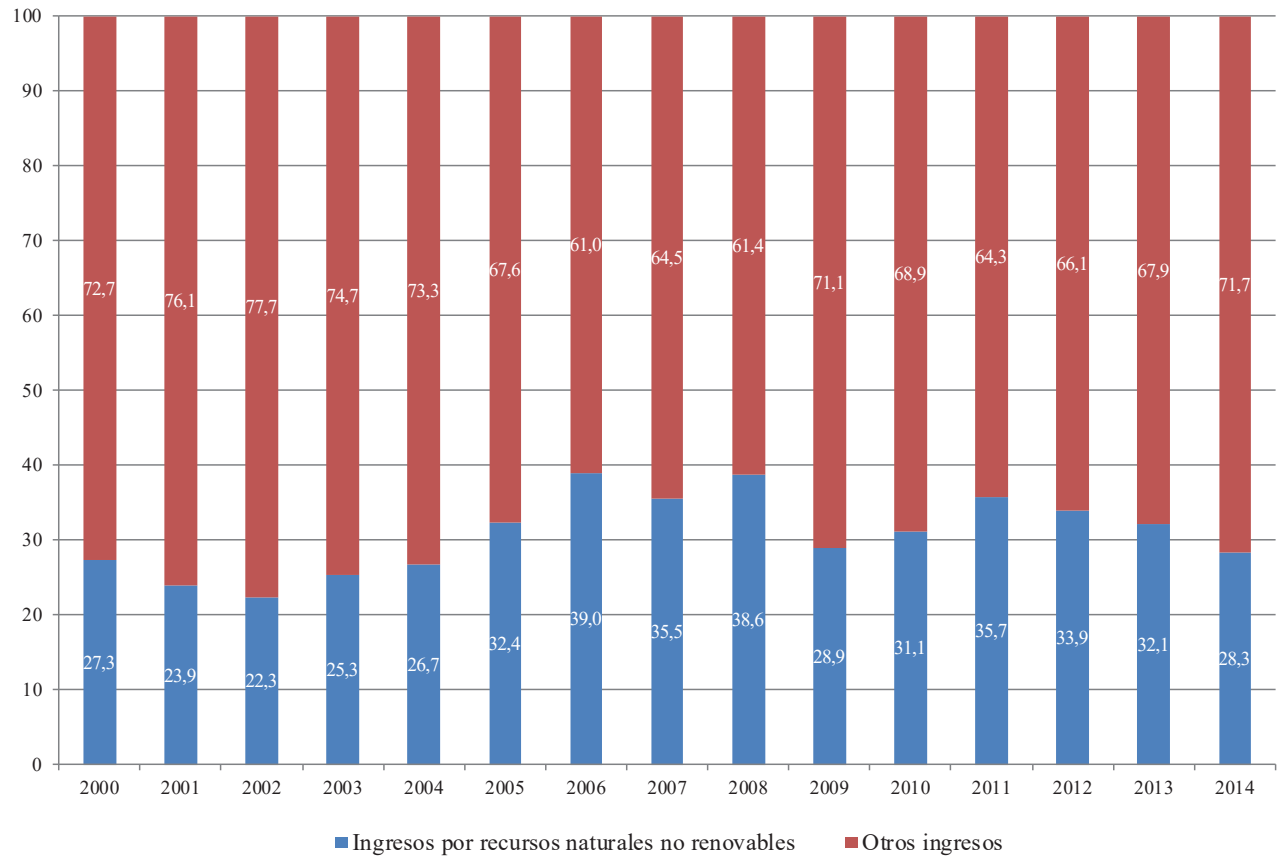

Fuente: Database BID-CIAT y CEPALSTAT. 
Participación relativa de los ingresos por recursos naturales no renovables sobre los ingresos fiscales totales

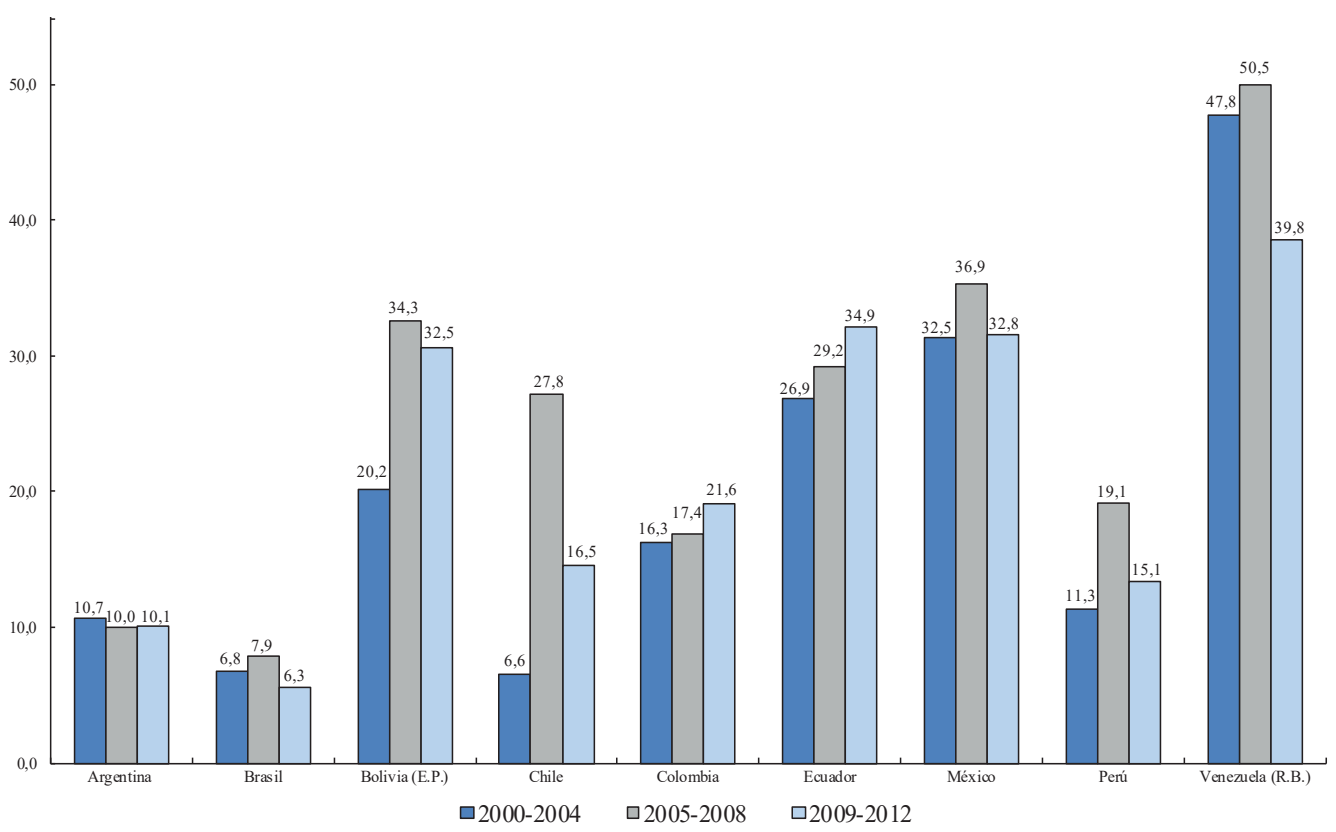

Fuente: OCDE, Cepal y CIAT (2013).

Gráfico 7.

Participación sobre el total y porcentaje del PIB de los ingresos fiscales de recursos naturales no renovables, 2014

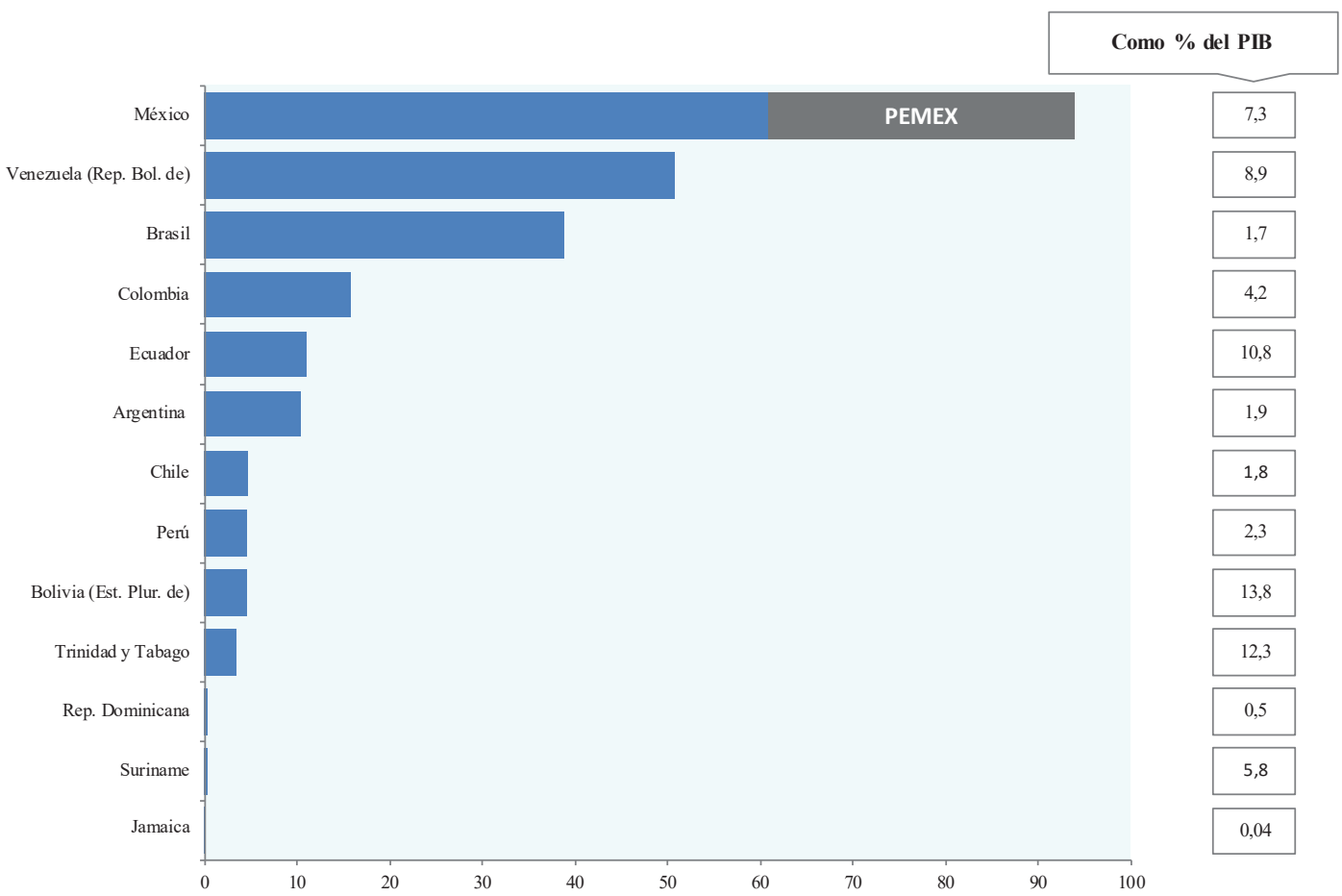

Fuente: OCDE, Cepal y CIAT (2016). 
de Venezuela, para el periodo 2005-2008, comprendieron más de la mitad de los ingresos totales.

En el gráfico 7 puede verse que, por ejemplo, para el caso de Bolivia, Venezuela y Ecuador, estos recursos han significado valores cercanos o superiores al $10 \%$ del PIB. En Argentina, la participación del 1,9\% del PIB incluye la recaudación por derechos de exportación sobre el agro, dado que, de acuerdo con la Cepal (2014a), y en forma similar a lo ocurrido en México, la producción de hidrocarburos y la consecuente exportación - sujeta a derechos de exportación- han registrado disminuciones.

\section{REVISIÓN DE LA LITERATURA SOBRE LA TEMÁTICA DE ESFUERZO FISCAL}

Una estimación de la base imponible potencial de los impuestos debería abarcar tanto los gastos tributarios como las distintas estimaciones sobre el nivel de evasión. Las dificultades metodológicas para la definición de las bases imponibles comparables internacionalmente, su administración y las deficiencias existentes en los correspondientes sistemas de estadísticas de cada país (Cetrángolo y Gómez Sabaini, 2009; Pecho, Peláezy Sánchez, 2012), además de la dificultas de armonizar las metodologías de cálculo, representan complejidades muy difíciles de superar para generar resultados comparables internacionalmente.

En la literatura, varios trabajos han avanzado en la estimación empírica tanto para países desarrollados como para algunos países en desarrollo, aunque para estos últimos -entre los que se cuentan los de América Latina-, los estudios se han focalizado en evaluar la magnitud de la evasión en el impuesto a la renta tanto de personas físicas como de sociedades (Jiménez, Gómez Sabaini y Podestá, 2010), el impuesto de valor agregado (IVA) (Pecho et al., 2012) o los aportes a los sistemas de seguridad social (Gómez Sabaini y Cetrángolo, 2014).

Una manera de sortear estos inconvenientes metodológicos descansa en las estimaciones de esfuerzo fiscal. Los gobiernos recaudan la mayor parte de sus ingresos desde el poder coercitivo; por supuesto, la capacidad tributaria de los países es útil solo cuando los gobiernos están dispuestos a cobrar impuestos en relación con esa capacidad. El esfuerzo fiscal de los gobiernos proporciona una medida del grado en que estos están dispuestos a gravar la capacidad en mención. Una medida del esfuerzo tributario, según Aronson y Hilley (1986), sería comparar la recaudación per cápita con el ingreso per cápita del país.

En línea con lo anterior, este artículo busca identificar factores que contribuyan a la recaudación fiscal en América Latina y el Caribe; en segunda instancia, los factores mencionados que influyen en el nivel de presión fiscal podrán ayudar a explicitar si hay espacio para incrementar los recursos generados por vía fiscal, en caso de que la recaudación potencial estimada difiriera en gran medida de la efectiva.

El presente apartado se dividirá en dos secciones: en primer lugar, se presentará una revisión de la literatura sobre la temática; luego se describirán las variables empleadas en la literatura, los datos utilizados, sus fuentes y los signos esperados a priori en la relación de causalidad planteada en forma preliminar.

\section{Antecedentes en la materia}

La mayoría de trabajos existentes en la literatura se focalizan en estimaciones sobre el potencial recaudatorio del sistema tributario en general, mientras algunos otros se focalizan en el impuesto a la renta o en los impuestos correspondientes a los niveles subnacionales de gobierno.

A grandes rasgos, podría establecerse que existen tres enfoques en la literatura para el cálculo del esfuerzo fiscal (Cyan, Martínez-Vázquez y Vulovic, 2013). En el primero de ellos -el enfoque tradicional de regresión-, el esfuerzo fiscal se mide al comparar la recaudación actual como porcentaje de la recaudación potencial. Pueden citarse varios antecedentes al respecto, como los realizados para países desarrollados (Kenney y Winner, 2006; 
Gordon y Li, 2009, entre otros) y algunos países en desarrollo (p. e., Davoodi y Grigorian, 2007).

Perry et al. (2006), en un enfoque cross section, encuentran que la recaudación efectiva de los países es inferior a la potencial en todos los impuestos, luego de relacionar la presión tributaria de cada país y el logaritmo del PIB per cápita; por ejemplo, para el caso del impuesto a la renta personal, Argentina recauda 4,4 puntos del PIB por debajo de su potencial, en tanto Brasil recauda 3,7 puntos del PIB.

Artana y Templado (2010) analizan en un enfoque cross section, para una muestra de 118 países y a través de un modelo lineal generalizado, si los países están recaudando por encima de su potencial. El ingreso per cápita, el grado de alfabetización, la participación de petróleo y minería en las exportaciones y el grado de monetización (M2) determinan positiva y significativamente la capacidad tributaria. Las variables de distribución del ingreso no resultan ser significativas, mientras que la variable dummy por país latinoamericano, el crecimiento poblacional y el ratio de agricultura a PIB impactan negativa y significativamente la determinación de la capacidad tributaria.

Al comparar los valores de carga tributaria predichos con los efectivos, los autores concluyen que Argentina recauda un $13 \%$ del PIB en exceso del valor pronosticado por el modelo, mientras que, para impuestos sobre ingresos y propiedad, el exceso es de alrededor de 6 puntos del PIB. Este resultado es el más alto para América Latina y uno de los más elevados en el ámbito internacional.

Stotsky y WoldeMariam (1997) utilizan un modelo de datos de panel con una muestra de 46 países del África subsahariana, con miras a analizar los determinantes del esfuerzo fiscal luego de relacionar el ratio de recaudación tributaria a PIB con un conjunto de variables explicativas (el ingreso per cápita, la participación del sector minero, industrial y agropecuario en el PIB, el ratio de exportaciones y de importaciones a PIB y los programas de asistencia financiera del FMI). El estudio concluye que la participación de la agricultura y de la minería en el PIB afecta negativa y significativamente la capacidad tributaria, mientras que el ingreso per cápita y el ratio de exportaciones a PIB afectan positiva y significativamente la capacidad de generación de recursos fiscales.

El índice de esfuerzo fiscal se calcula al comparar la recaudación actual y la predicha según el modelo; como resultado, arroja que la mayoría de los países del África Subsahariana presentan exceso de tributación. Sin embargo, los países con índices bajos deberían poner énfasis en aumentar la recaudación, y no en una reducción de los gastos ante un déficit presupuestario.

Gupta (2007) utiliza un panel de 105 países en desarrollo por un periodo de 25 años $^{4}$. El ingreso per cápita y la apertura económica afectan positiva y significativamente todas las regresiones de efectos aleatorios y la mayoría de las especificaciones de efectos fijos a la recaudación potencial, mientras que el impacto es positivo y significativo si se considera la participación de la agricultura, y positivo y no significativo para los factores institucionales. Argentina, Costa Rica, Letonia, Lituania y Eslovaquia se encuentran entre los países que tienen una recaudación inferior al potencial.

El segundo de los enfoques subraya que el rol de las instituciones puede agregarse a la lista de determinantes del esfuerzo fiscal (Bird, MartínezVázquez y Torgler, 2008); el nivel de recaudación tributaria puede, en este caso, estar afectado por la calidad de los servicios públicos, la gobernanza y las instituciones estatales.

Mediante un modelo de datos de panel para América Latina en el periodo 1990-2008, Jiménez, Gómez Sabaini y Rossignolo (2012) y Rossignolo (2012) estiman la capacidad tributaria de los países para predecir el esfuerzo tributario en relación con el impuesto a la renta total y de las personas físicas, en una muestra de 15 países. Los signos encontrados en las estimaciones coinciden con lo esperado: relación positiva de la recaudación de ambos impuestos con el PIB per cápita; así también,

$4 \quad$ Davoodi y Grigorian (2007) analizan el caso de Armenia y enfatizan el tratamiento de la variable de moral tributaria. 
la recaudación crece a mayor desigualdad en la distribución del ingreso, educación y grado de institucionalidad.

A partir de las predicciones del modelo, se concluye que a partir del 2000 los países de América Latina se acercan a su potencial recaudatorio; para personas físicas durante esta década se presentan valores cercanos o inferiores a 1, mientras que, para el total del impuesto, la recaudación efectiva excede a la potencial, aunque decrece al aproximarse al final de la década.

En consecuencia, existen casos de países con elevada presión tributaria, como Brasil, México y Perú, donde se está muy cerca o por encima del nivel de recaudación potencial. En otros casos, como Argentina y Chile, en el impuesto a la renta de personas físicas se está por debajo del potencial, pese a estar en el total del impuesto en un nivel próximo al potencial. Esto implica un desbalance en la composición del impuesto.

Más recientemente, algunos autores han utilizado el análisis de frontera estocástica para computar el esfuerzo fiscal, con el propósito de identificar la ineficiencia en la recaudación de impuestos. Pessino y Fenocchietto (2010, 2013) utilizan un modelo de frontera impositiva para representar el máximo nivel de imposición alcanzable a partir de un conjunto de variables explicativas. En este modelo, el esfuerzo fiscal debe medirse en relación con el de los demás países, lo cual no necesariamente implica que el país sea ineficiente en su recaudación de impuestos.

A través de un modelo de datos en panel, se explica la recaudación tributaria a PIB en el grado de desarrollo económico, el valor agregado de la agricultura al PIB, el nivel de educación, el grado de apertura económica, la desigualdad en la distribución del ingreso, la inflación y las ineficiencias en la recaudación (índice de transparencia).

Los autores encuentran una relación positiva y significativa entre la presión tributaria y el grado de desarrollo económico, educación y apertura económica, y una relación negativa para la desigualdad en la distribución del ingreso, la ineficiencia en la recaudación y la participación de la agricultura.

Garg, Goyal y Pal (2014) realizan una estimación reciente para el caso de los estados subnacionales en la India, enfatizan el rol de las instituciones políticas y la economía política de las transferencias a los niveles subnacionales de gobierno. Cyan et al. (2013) hacen una comparación entre las estimaciones, tomando como variable dependiente la relación entre impuestos y gasto público.

Este trabajo se enmarca en esta última de las alternativas. El propósito es utilizar el análisis de frontera estocástica, con el fin de computar el esfuerzo fiscal de los países de América Latina; la metodología de estimación se desarrollará a partir de un modelo de datos de panel. A la manera de Pessino y Fenocchietto (2010, 2013), Cyan et al. (2013) o Garg et al. (2014), entre otros, se procurará estimar, en primera instancia, la capacidad de las economías latinoamericanas para generar un nivel de tributación en función de diversas variables explicativas $^{5}$. El periodo analizado es 1990-2013 para 23 países de América Latina y el Caribe; las fuentes de información empleadas son la base de datos del proyecto IDLA, BID-CIAT y CEPALSTAT ${ }^{6}$.

En segundo lugar, una vez estimados los coeficientes del modelo, se generará una capacidad fiscal predicha y se relacionará esta con la presión fiscal de cada momento, para obtener una estimación de esfuerzo fiscal que determine si los países están recaudando cerca de su potencial. La brecha entre la recaudación observada y la frontera establece el grado de desaprovechamiento tributario o el grado de ineficiencia.

\section{CONSIDERACIONES PRELIMINARES Y MODELO TEÓRICO}

El objetivo de este trabajo es analizar la capacidad de generar crecimiento recaudatorio en la

\footnotetext{
5 Las estimaciones fueron realizadas mediante Stata 13.

6 Véanse, respectivamente, estos enlaces: https://www.wider. unu.edu/project/wiid-world-income-inequality-database y https://www.ciat.org/recaudacion/
} 
denominada carga fiscal ajustada. Así, de acuerdo con Barreix et al. (2013), se incluyen como ingresos fiscales tanto los recursos tributarios como los provenientes de la seguridad social y otros ingresos, al igual que los originados de la explotación de recursos naturales y todos los aportes obligatorios realizados para financiar al sistema de seguridad social, incorporando aquellos que las definiciones internacionales vigentes habitualmente excluyen. Esta metodología se realiza con el fin de compatibilizar los ingresos fiscales de todos los países, considerando la heterogeneidad de las fuentes de recursos y de los arreglos institucionales correspondientes, dado que existen ingresos provenientes de diversas fuentes que complementan a los ingresos exclusivamente tributarios ${ }^{7}$.

En los anexos 1 y 2 se muestran los estadísticos descriptivos y las fuentes de información de las variables por considerar; cabe consignar que se han adicionado a las variables explicativas algunas variables de transparencia e institucionales; por ejemplo, se ha utilizado un indicador de estabilidad gubernamental y otro de corrupción (Worldwide Governance Indicators) ${ }^{8}$.

\section{Algunas relaciones a priori entre las variables empleadas}

En los gráficos 8 a 13 se observa la correlación entre la presión fiscal y las distintas variables explicativas, tanto para el caso general como para el indicador de carga fiscal ajustada, en un análisis no condicionado

7 En este caso, se considerarán las contribuciones a la seguridad social como parte de la recaudación, de acuerdo con Cepal (2006). Allí se especifica que si los aportes son obligatorios, el sistema está a cargo de instituciones públicas y las prestaciones cumplen una función redistributiva, las cargas salariales se deben considerar como parte de la presión tributaria.

8 En otros estudios se utilizan indicadores adicionales como globalización, violencia social o política, etc. La adición de estas variables al modelo habría significado una reducción considerable en la cantidad de datos con los que se realizaron las estimaciones (dado que no se cuenta con valores de estas para todos los países y periodos analizados), además de sobredeterminar los modelos por la gran cantidad de variables explicativas incluidas en relación con la cantidad de observaciones. y en promedios, para cada país, de todos los periodos analizados. En el gráfico 8 puede apreciarse que existe una correlación positiva entre la presión tributaria (ingresos tributarios con respecto al PIB) y el ingreso per cápita, lo que es consistente con el resto de la literatura. Para el ratio de carga fiscal ajustada, la correlación es de 0,46 , significativa al 2,5\%, mientras que la presión tributaria, incluyendo seguridad social, si bien es positiva $(0,22)$, es poco significativa $(0,31)$.

La correlación negativa existente entre la participación de la agricultura en el producto, tanto para la recaudación total como para la carga fiscal ajustada, es también coincidente con los antecedentes empíricos; aunque en el caso de esta última la relación negativa es más marcada y significativa que en el primer caso $(-0,39$ y $-0,55$, respectivamente, significativas al $5 \%$ y $1 \%$, respectivamente $)^{9}$.

Se puede apreciar una correlación positiva entre la recaudación tributaria total y el índice de Gini de desigualdad en la distribución del ingreso, sin signo definido con la carga ajustada ${ }^{10}$. Sin embargo, ninguna de las correlaciones es significativa.

Sin embargo, las correlaciones son muy superiores si se consideran los promedios por año para todos los países. Al analizar el producto per cápita, la correlación es de 0,98; por otro lado, considerando el índice de Gini, la correlación es negativa y superior al 0,60 para ambas variables, mientras que la correlación con la agricultura es negativa y superior a 0,86 , siendo significativa en todos los casos.

9 Son numerosos los estudios que encuentran una relación negativa y significativa entre la participación de la agricultura y la recaudación tributaria; entre otros, Stotsky y WoldeMariam (1997), Piancastelli (2001), Bird et al. (2004), Gupta (2007) y Pessino y Fenochietto (2010).

10 No existe consenso en la literatura en función del signo de la relación entre la desigualdad de la distribución del ingreso y la recaudación tributaria. Entre los estudios que encuentran una relación negativa, si bien controlando por otras variables, cabe destacar Bird, Martínez-Vázquez y Torgler (2004) y Pessino y Fenochietto (2010). 
Grafico 8 .

Correlación entre la presión fiscal promedio y el PIB per cápita (carga fiscal ajustada)

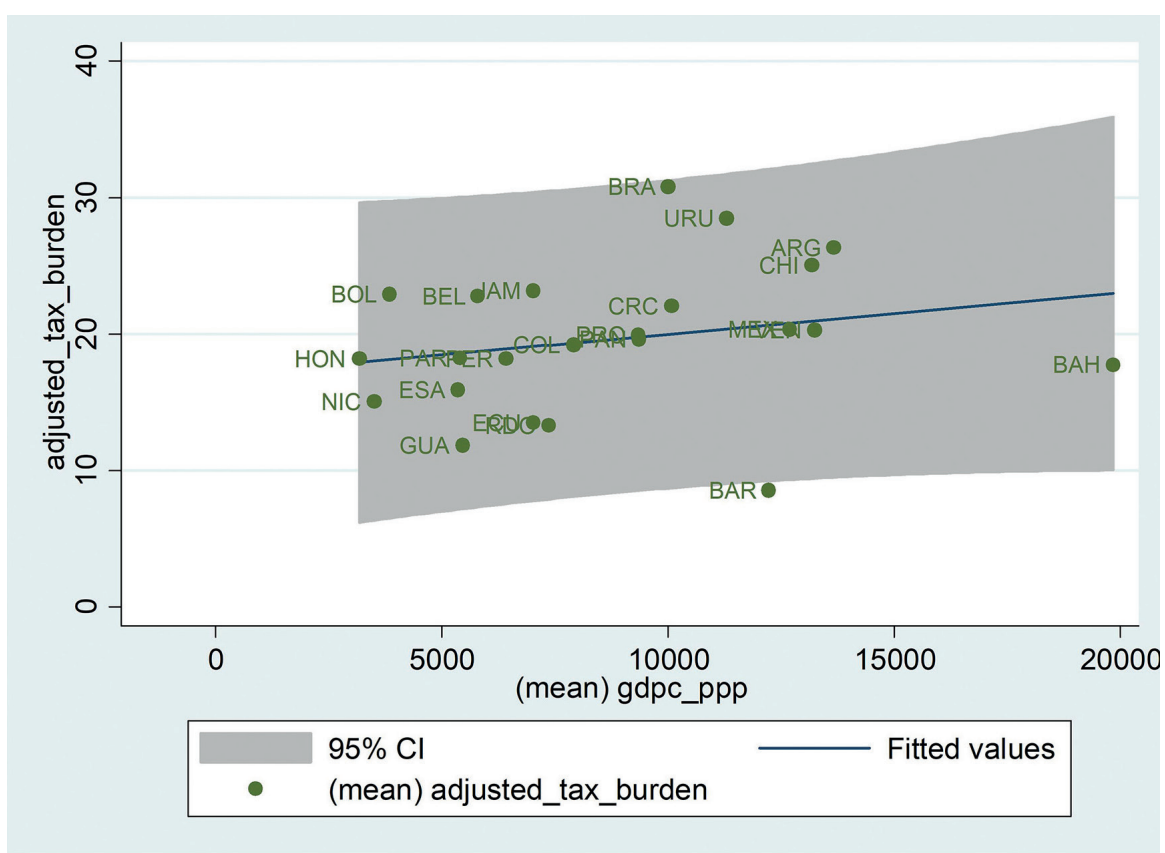

Fuente: cálculos del autor basados en datos de BID-CIAT y CEPALSTAT.

Gráfico 9.

Correlación entre la presión fiscal promedio y el PIB per cápita (presión tributaria incluyendo seguridad social)

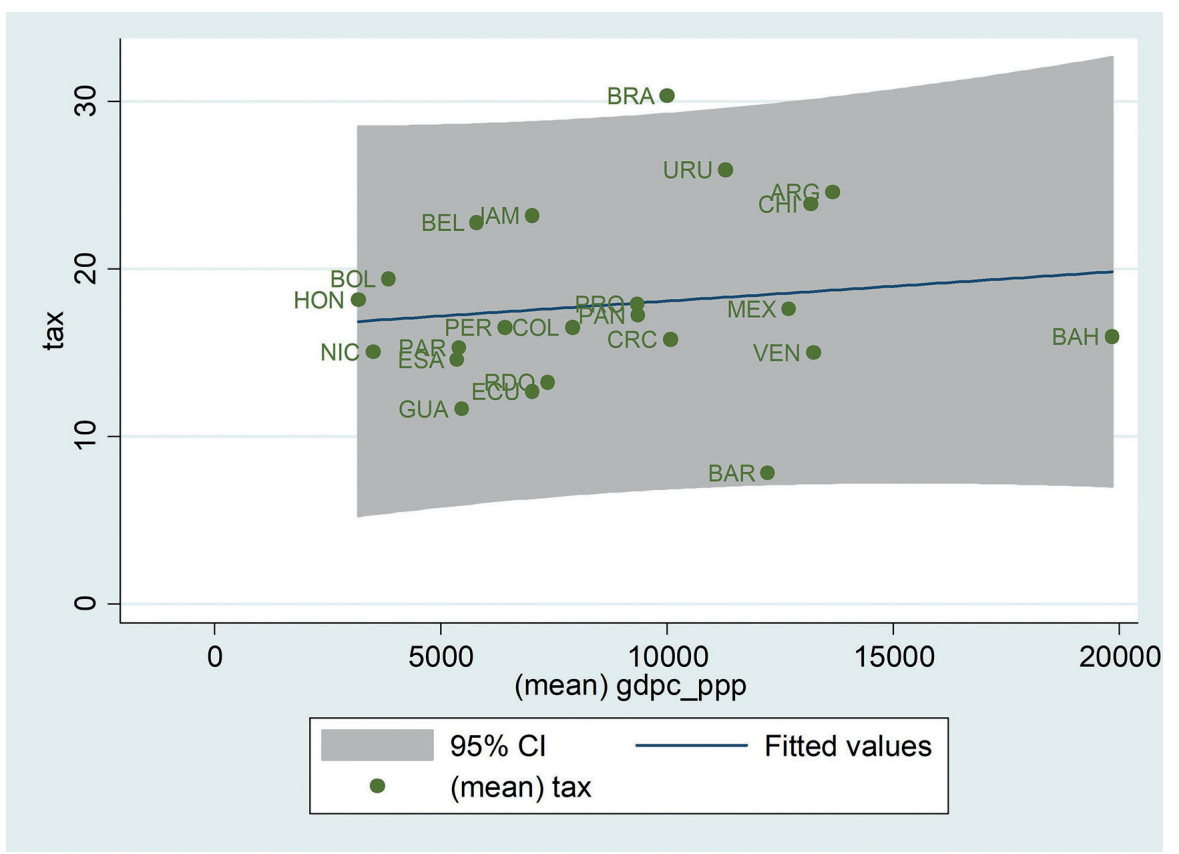

Fuente: cálculos del autor basados en datos de BID-CIAT y CEPALSTAT. 
Gráfico 10.

Correlación entre la presión tributaria promedio y la participación de la agricultura (carga fiscal ajustada)

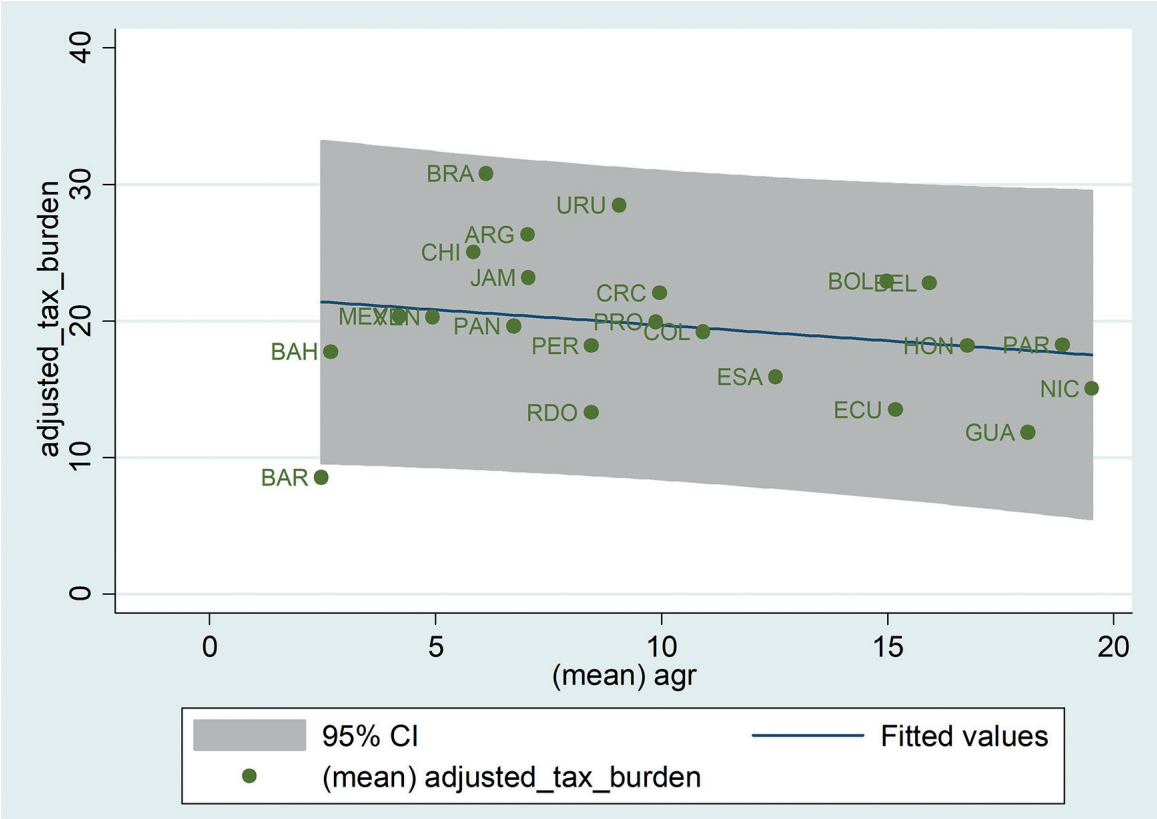

Fuente: cálculos del autor basados en datos de BID-CIAT y CEPALSTAT.

Gráfico 11 .

Correlación entre la presión tributaria promedio y la participación de la agricultura (presión tributaria incluyendo seguridad social)

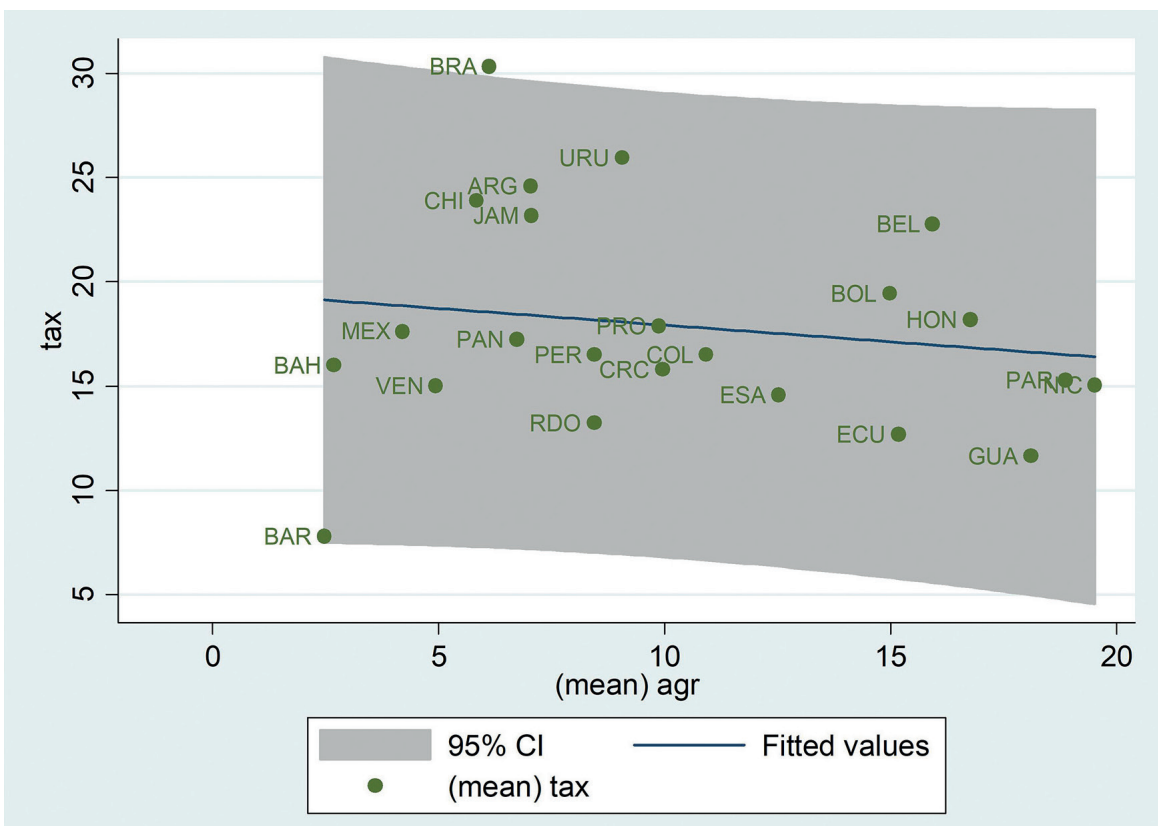

Fuente: cálculos del autor basados en datos de BID-CIAT y CEPALSTAT. 
Gráfico 12.

Correlación entre la presión fiscal promedio y el índice de Gini (carga fiscal ajustada)

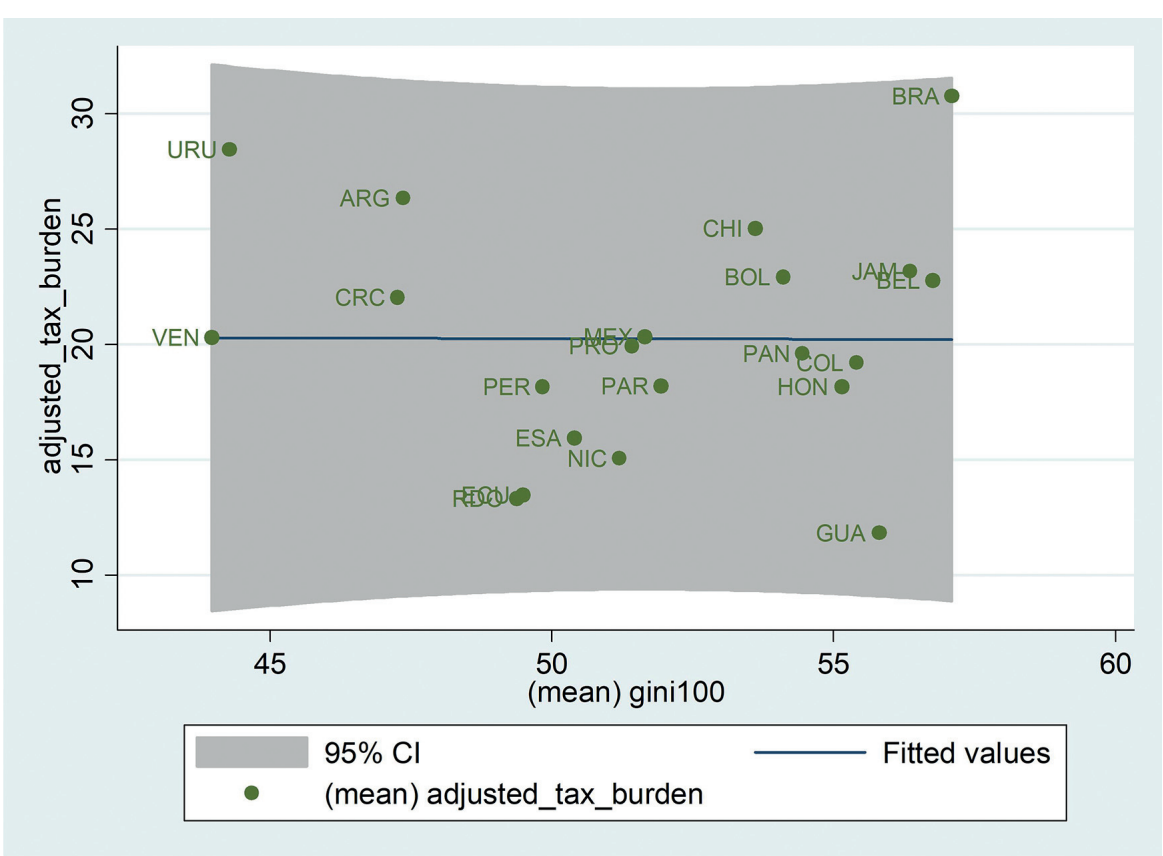

Fuente: cálculos del autor basados en datos de BID-CIAT y CEPALSTAT.

Gráfico 13.

Correlación entre la presión fiscal promedio y el índice de Gini (presión tributaria incluyendo seguridad social)

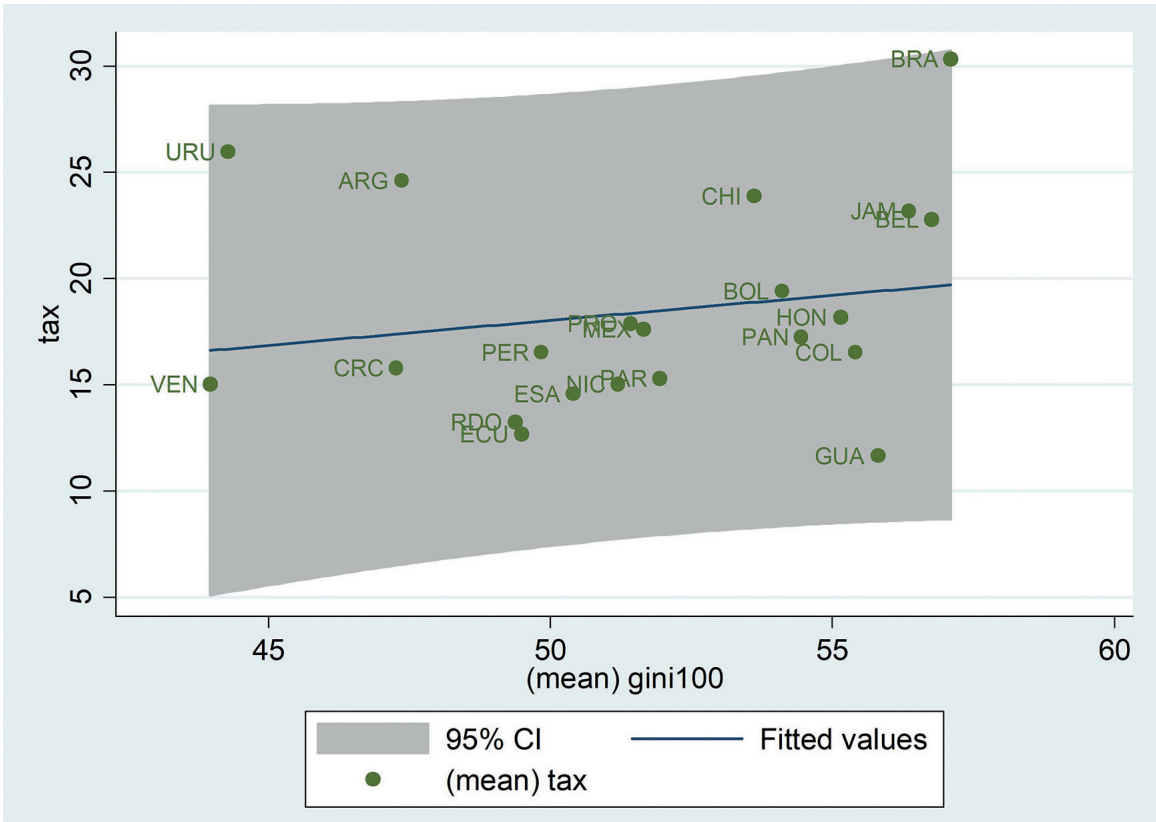

Fuente: cálculos del autor basados en datos de BID-CIAT y CEPALSTAT. 


\section{Aspectos teóricos}

En esta sección se presentará la metodología empleada en el análisis de la explicación sobre los determinantes del esfuerzo fiscal de los países, para luego estudiar el comportamiento de la recaudación efectiva en relación con su nivel potencial.

Los modelos de frontera estocástica de producción (SFA) constituyen una extensión del análisis de regresión. De manera similar al caso de las funciones de producción, el modelo de frontera estocástica impositiva tributaria mide el máximo nivel de recaudación que una unidad de gobierno puede alcanzar a partir de una serie de factores determinantes. La brecha entre la recaudación efectiva y la máxima alcanzable indica tanto la ineficiencia recaudatoria técnica como cuestiones de economía política asociadas a la recaudación propiamente dicha. Los insumos que contribuyen a la construcción de las fronteras tributarias son menos evidentes que en el caso de las funciones de producción, porque si bien el producto per cápita o el nivel de educación pueden ser determinantes de la recaudación, en el caso de otros insumos, como la desigualdad o la inflación, el efecto no es claro, con lo cual podrían estar afectando la propia eficiencia recaudatoria y no la frontera (Pessino y Fenocchietto, 2010).

Las fronteras de producción estocásticas fueron planteadas inicialmente por Aigner, Lovell y Schmidt (1977). La especificación original es una función de producción para un análisis de sección cruzada; el término de error, a diferencia de una regresión convencional, tiene dos componentes: un componente aleatorio y otro de eficiencia técnica:

$Y_{i}=x_{i} b+\left(V_{i}-U_{i}\right), i=1, \ldots, N$

En este caso, $Y_{i}$ representa (el logaritmo de) la producción de la iésima unidad; $x_{i}$ es un vector $k^{\prime} 1$ de insumos; $b$ es un vector de parámetros desconocidos; $V_{i}$ son variables aleatorias iid. $N\left(0, s_{v}{ }^{2}\right)$ e independientes de los $U_{i,}$ que son variables aleatorias no negativas que representan la ineficiencia técnica y son iid $\mathrm{N}\left(0, \mathrm{~s}_{\mathrm{u}}{ }^{2}\right) \mid$. En el caso de este modelo, $Y_{i}$ representaría el máximo nivel de recaudación alcanzable.

Algunas variaciones de este modelo han sido aplicados en la literatura con diferentes estructuras del término de error y diferentes hipótesis sobre las distribuciones del mismo.Battese y Coelli (1992) presentan un modelo de frontera estocástica para datos de panel (aplicable también a paneles no balanceados) con efectos por firma que, se asume, se distribuyen como variables normales truncadas, que pueden variar sistemáticamente en el tiempo.

$Y_{i t}=x_{i t} b+\left(V_{i t}-U_{i t}\right), i=1, \ldots, N, t=1, \ldots, T$

En este caso, las variables se definen de la misma manera que en [1], agregando la variabilidad en $t$. Las $V_{i t}$ son aleatorias iid $N\left(0, \mathrm{~s}_{\mathrm{v}}{ }^{2}\right)$, independientes de los $U_{i t}=\left(U_{i} \exp (-\mathrm{h}(\mathrm{t}-\mathrm{T}))\right)$. Los $U_{i}$ son no negativos, que representan la ineficiencia técnica en la producción, y se suponen variables iid truncadas en cero de una distribución $N\left(\mathrm{~m}, \mathrm{~s}_{\mathrm{U}}{ }^{2}\right)$, en tanto $h$ es un parámetro por estimar. Si $h$ es 0 , la ineficiencia es invariante en el tiempo; si el valor del parámetro es positivo, las ineficiencias decrecen en el tiempo; mientras que si $T=1$, corresponde al modelo original de sección cruzada seminormal de Aigner, Lovell y Schmidt (1977).

De acuerdo con Battese y Corra (1977), es posible reemplazar $s_{V}{ }^{2}$ y $s_{U}{ }^{2}$ por $s^{2}=s_{V}{ }^{2}+s_{U}{ }^{2}$ y $g=s_{U}{ }^{2}$ / $\left(s_{v}{ }^{2}+s_{u}{ }^{2}\right)$ (este valor debe estar entre 0 y 1 ) y $\lambda=s_{u}$ / $s_{V}$ (pudiendo adoptar cualquier valor no negativo). Cuando $g$ tiende a $0, s_{V}{ }^{2}$ tiende a infinito o $s_{u}{ }^{2}$ tiende a 0 , con lo cual el modelo se puede estimar por mínimos cuadrados ordinarios; si $g$ tiende a 1 , el modelo genera una frontera determinística sin ruido aleatorio. Mientras tanto, $\lambda$ provee información sobre la contribución relativa de ambos componentes de error sobre el error total.

Además, es importante analizar si, además de analizar los determinantes de la frontera recaudatoria, existen factores que afecten la eficiencia propiamente dicha. Algunos estudios empíricos estimaron fronteras estocásticas de producción (o su inversa, de costos) y luego llevan a cabo una regresión de las predicciones de los niveles de eficiencia establecidos en el paso anterior tomando variables 
específicas de las firmas como variables independientes, con el objetivo de identificar factores que afectan la eficiencia. Como estos procedimientos de dos etapas no generan estimadores eficientes, Battese y Coelli (1995) proponen para estimar en una sola etapa el siguiente modelo:

$Y_{i t}=x_{i t} b+\left(V_{i t}-U_{i t}\right), i=1, \ldots, N, t=1, \ldots, T$

Aquí las $V_{i t}$ son iid. $N\left(0, \mathrm{~s}_{\mathrm{v}}{ }^{2}\right)$ e independientes de los $U_{i t}$ que son no negativos distribuidos como una normal truncada en $\mathrm{ON}\left(\mathrm{m}_{\mathrm{it}} \mathrm{s}_{\mathrm{u}}{ }^{2}\right)$ considerando:

$m_{i t}=z_{i t} d$,

En este caso, $z_{i t}$ es un vector $p^{\prime} 1$ de variables independientes que pueden afectar la eficiencia, $y$ $d$ is un vector 1 ' $p$ de parámetros por estimar. La especificación del modelo abarca a un conjunto de otras representaciones como casos especiales. Si se define $T=1$ y $_{\text {it }}$ contuviera el valor 1 y ninguna otra variable (solo una constante), el modelo se reduce a la especificación normal truncada en Stevenson (1980), donded (el único elemento en d) tiene la misma interpretación que el parámetro $m$ en Stevenson (1980).

Los modelos definidos en [3] y en [4] no tienen al modelo definido en [2] como un caso especial, y a la inversa tampoco dado que estas especificaciones no están anidados, con lo cual no puede definirse un conjunto de restricciones que permita testear entre los modelos alternativos.

A partir de la especificación del modelo, los valores de los parámetros permitirán testear las siguientes hipótesis:

La ineficiencia técnica no es afectada por las variables explicativas $(\delta=0)$.

El término de ineficiencia técnica no es estocástico, con lo cual $\lambda=0$, estando $\lambda$ expresado como el ratio de la desviación estándar del término de ineficiencia con relación a la desviación estándar del término de error (como se expresara con anterioridad).

La construcción del término de error es la diferencia conceptual entre las estimaciones de los modelos de regresión — presentados en la sección sobre revisión de la literatura-y los modelos SFA, de frontera estocástica. En los modelos de regresión, el término de error, que representa la ineficiencia, puede ser positivo o negativo, lo cual indica que una región puede desviarse de su recaudación predicha promedio; por el contrario, en los modelos SFA, el componente no negativo del término de error asegura que la recaudación observada nunca puede exceder a la óptima (Pessino y Fenochietto, 2010, 2013; Cyan et al., 2013; Garg et al., 2014).

En términos formales, los modelos serían los siguientes:

OLS: $q_{i}=b_{0}+b_{1} x_{i}+v_{i}$

Determinística : $\mathrm{q}_{\mathrm{i}}=\mathrm{b}_{0}+\mathrm{b}_{1} \mathrm{x}_{\mathrm{i}}-\mathrm{u}_{\mathrm{i}}$

SFA: $q_{i}=b_{0}+b_{1} x_{i}+v_{i}-u_{i}$

Donde $v_{i}$ es el "ruido" término de error simétrico (p. e., distribución normal) y $u_{i}$ es el "término de error de ineficiencia", no negativo (p. e., half-normal distribution). El modelo que se ha de estimar es el siguiente:

$\operatorname{lnt}_{i t}=a+b^{\top} x_{i t}+v_{i t}-u_{i t}$

En consecuencia, se define el esfuerzo fiscal como el cociente entre la presión fiscal observada y la correspondiente frontera recaudatoria estocásti$\mathrm{ca}$, con valor comprendido entre 0 y 1 . La diferencia entre la recaudación observada y la frontera puede ser interpretada como el impuesto no recaudado. Esto no necesariamente corresponde a ineficiencias en la recaudación de impuestos, sino al hecho de que las sociedades hayan decidido que la recaudación sea baja por preferencias en la provisión de bienes públicos también reducida (Pessino y Fenochietto, 2010). La fórmula es:

$$
\begin{aligned}
T E_{i}= & \frac{q_{i t}}{\exp \left(x_{i t}^{\prime} \beta+v_{i t}\right)}= \\
& \frac{\exp \left(x_{i t}^{\prime}+v_{i t}-u_{i t}\right)}{\exp \left(x_{i t}^{\prime}+v_{i t}\right)}=\exp \left(-u_{i t}\right)
\end{aligned}
$$


En [9], $T E_{i}$ representa el esfuerzo fiscal, calculado como cociente entre la carga fiscal observada (numerador de la expresión) y la capacidad recaudatoria (el denominador). Por construcción del modelo, la capacidad fiscal es siempre mayor o igual a la recaudación efectiva, con lo cual en el modelo se evalúa cuán cerca se está del valor del potencial recaudatorio.

\section{ESTIMACIÓN DE LOS DETERMINANTES DEL ESFUERZO FISCAL}

El propósito de esta sección es delinear la metodología empírica empleada en el análisis. Se estimar, entonces, el esfuerzo fiscal de cada país en relación con la carga fiscal ajustada ${ }^{11}$. La variable dependiente es el cociente de los ingresos fiscales ajustados sobre el PIB (en logaritmos).

\section{Variables consideradas}

En muchos casos, tal como explican Pessino y Fenochietto (2010), no está claramente especificado cuáles variables forman parte de los determinantes de la recaudación tributaria y cuáles intentan explicar la ineficiencia. Es evidente, por ejemplo, que el PIB per cápita u otros indicadores económicos, institucionales o demográficos, como el nivel de educación, pueden ser considerados como inputs de la recaudación. En otros casos, como la inflación o la corrupción, pueden ser considerados como inputs o afectan directamente a la ineficiencia. Una descripción de la relación esperada y su signo se exponen a continuación.

a) Potencial recaudatorio. En la identificación de los determinantes del potencial,

11 Dado que, como se mostró en la revisión de la literatura sobre la temática de esfuerzo fiscal, las correlaciones de varias variables explicativas con relación a la carga fiscal ajustada y a la recaudación tributaria no difieren en gran medida, por razones de espacio en este trabajo se presentarán las estimaciones de la capacidad fiscal relacionada con la primera de las mencionadas. Los resultados en relación con la recaudación tributaria, incluyendo seguridad social, no difieren significativamente de los aquí presentados y se pueden solicitar al autor. la hipótesis conducente es que la capacidad recaudatoria depende de factores económicos y demográficos:

- Ingreso per cápita (PPP 2005). La variable explicativa más utilizada es el nivel de desarrollo económico, medido a través del ingreso por habitante. La idea subyacente es que los países con mayor nivel de ingreso demandarán mayores bienes públicos y estarán dispuestos a pagar mayores impuestos para financiarlos; sin embargo, el hecho de que un aumento del ingreso genere que el mismo nivel de recaudación pueda alcanzarse con una alícuota más baja determina que el efecto del nivel de renta sobre la tasa impositiva sea incierto. No obstante, la mayor parte de la evidencia empírica sugiere que el primer efecto domina al segundo, con lo cual el signo esperado es positivo (Pessino y Fenochietto, 2010, 2013).

- El grado de apertura de la economía. De acuerdo con Gupta (2007), el efecto de la apertura económica en los ingresos tributarios puede ser ambiguo. Una reducción de los aranceles al comercio exterior generaría una disminución en los recursos fiscales, los que solo podrían ser incrementados si el aumento en importaciones y exportaciones ocasionara también un incremento en las bases imponibles de los impuestos tradicionales (efecto de curva de Laffer), como el IVA a las importaciones o el impuesto a la renta, al eliminar los derechos de exportación.

- La incorporación de sectores económicos difíciles de gravar. La variable explicativa empleada es el valor agregado por el sector agropecuario en porcentaje del PIB de cada país ${ }^{12}$. Este sector resulta

12 Algunos estudios incluyen como variable explicativa la participación de la industria en el PIB. A la inversa del sector agropecuario, más difícil de gravar, la presencia de un sector industrial más significativo debería relacionarse con un mayor grado de cumplimiento tributario. Las estimaciones realizadas en este caso no encuentran que la variable resulte 
complejo de gravar debido a que no solo está compuesto en una importante proporción por productores pequeños (Pessino y Fenochietto, 2010), sino que, además, en el caso de productores grandes, existen economías de escala en las concentraciones económicas que favorecen la evasión impositiva, por lo cual el signo esperado es negativo. Esto puede implicar tanto que el sector agropecuario sea difícil de gravar porque buena parte de él corresponde a subsistencia (o por evasión), como que estos sectores requieran de menor provisión de bienes públicos por estar basados en áreas rurales (Gupta, 2007).

- Nivel de educación. El efecto esperado, medido a través de la participación del gasto educativo en el PIB de cada país, es en general ambiguo (Cyan et al., 2013). Por un lado, una población con mayor nivel educativo puede comprender mejor las razones de por qué es relevante pagar impuestos, dado que de esa manera se puede financiar un mayor nivel de provisión de bienes públicos. Por otro lado, el mayor nivel educativo de la población podría otorgarle acceso a herramientas más sofisticadas para evitar el cumplimiento de sus obligaciones tributarias, lo que implicaría, a la inversa de la justificación anterior, una relación negativa.

- Desigualdad en la distribución del ingreso medida a través del índice de Gini. Los estudios encuentran que esta tiene un efecto negativo en la recaudación de impuestos (Bird, Martínez-Vázquez y Torgler, 2004; Gupta, 2007; Pessino y Fenochietto, 2010). Se considera que una desigualdad creciente reduce la base imponible a través de varios canales

significativa (los resultados pueden solicitarse al autor) Lamentablemente, la informalidad no pudo ser incluida como variable en el modelo, por no contarse con datos para todos los países y periodos analizados.
(Cyan et al., 2013); por ejemplo, en una sociedad más desigual, la administración tributaria depende en su mayoría de los sectores de ingresos más elevados para la recaudación de impuestos, lo que reduce la base tributaria. Una mayor desigualdad en la distribución del ingreso puede ocasionar un sector informal de mayor tamaño ${ }^{17}$, lo que también impacta negativamente en la recaudación. Adicionalmente, como argumento de política económica puede citarse que la desigualdad en la distribución también representa sectores concentrados cuya predisposición a evadir impuestos es elevada. Sin embargo, podría ocurrir que con mínimos impositivos constantes, una mayor desigualdad también incremente la base imponible de los impuestos.

b) Explicación de la ineficiencia técnica. Si bien, como se especificó, la literatura en la materia no es concluyente para determinar las variables determinantes de la ineficiencia, en este trabajo se ha elegido incluir las siguientes:

- Corrupción. Esta puede incrementar la ineficiencia técnica en el sistema tributario al introducir una inestabilidad permanente en el sistema político (Cyan et al., 2013). La variable empleada, control de la corrupción, implica que mayores valores se relacionan con una menor corrupción, con lo cual se espera una relación negativa entre esta variable y la ineficiencia técnica.

- Inflación. Medida a través del porcentaje de cambio en el índice de precios al consumidor, el efecto de la inflación en la recaudación de impuestos es ambiguo (Cyan et al., 2013). Por un lado, debido al efecto Olivera-Tanzi de rezago fiscal, la inflación decrece el valor real de los ingresos tributarios. En este sentido, los países que obtienen una gran parte de sus ingresos fiscales mediante emisión monetaria tienen una mayor ineficiencia 
en la recaudación de impuestos. Según estas explicaciones, cabría esperar una relación negativa; sin embargo, la eficiencia podría crecer con la inflación principalmente en el caso de los impuestos progresivos, sobre todo si se considera el hecho de que las autoridades fiscales no indexen adecuadamente las tasas de los impuestos y los tramos en los que se divide la base imponible.

- Complejidad del sistema tributario. Medido a partir del índice de Herfindahl sobre la estructura tributaria de cada país. La hipótesis subyacente es que un sistema tributario más simple genera una mayor facilidad para los contribuyentes de percibir el costo real del gobierno; de ahí que sea más probable que los gobiernos tengan menores gastos $y$, en consecuencia, menores recursos tributarios. Conversamente, sistemas tributarios más complejos llevan a gobiernos de mayor tamaño, con mayores niveles de gasto público $y$, consecuentemente, mayores impuestos para financiarlos y mayor eficiencia en la recaudación de impuestos.

Cabe consignar que sería importante poder agregar otras variables adicionales, como las de moral tributaria, que dependen de la información brindada a los contribuyentes sobre en qué se gasta, cuál es la efectividad del gasto (resultados), etc., o alguna variable relacionada con la facilidad para pagar impuestos; sin embargo, no pudo disponerse de ellas para todos los periodos y países analizados.

\section{Especificaciones de los modelos empleados y resultados de las estimaciones}

La adición de variables explicativas al modelo está determinada tanto por la significatividad individual de los coeficientes de estas como de cada modelo en su conjunto (test de Wald) y de la convergencia de las funciones de verosimilitud de cada estimación. Se adicionó una variable dummy para identificar outliers en cada una de las series de las variables dependientes; así también, debido al hecho de que no se rechaza la hipótesis de ausencia de heterocedasticidad, los errores estándares son robustos con clústeres en el ámbito de individuo.

Se estimó el modelo de Battese y Coelli (1992), donde se asume que la ineficiencia decae a una tasa constante con distribución normal con media cero (half normal); de igual manera, la especificación de Battese y Coelli (1995), con el término de ineficiencia siguiendo una distribución normal truncada y especificando variables que afectan la eficiencia (environmental variables) ${ }^{13}$ para diferentes conjuntos de variables explicativas.

Las variables independientes que explican la presión tributaria ajustada son el ingreso per cápita, el porcentaje del valor agregado por la agricultura, la apertura económica, el gasto público en educación y la desigualdad medida a través del índice de Gini. La complejidad del sistema tributario, la inflación y el indicador de corrupción son utilizados para explicar la ineficiencia (Pessino y Fenochietto, 2010).

En la tabla 1 se presentan los resultados de las estimaciones por máxima verosimilitud de los parámetros de la frontera estocástica recaudatoria. El ingreso per cápita afecta positivamente la recaudación tributaria; la participación de la agricultura impacta en forma negativa (sector más propenso a evadir impuestos); la apertura económica, de acuerdo con la literatura en la temática, genera resultados inconcluyentes; el gasto en educación, indicador de una mayor moral tributaria, afecta positiva y significativamente en todas las especificaciones.

El parámetro eta resulta positivo y significativo, con lo cual no se rechaza la hipótesis de que la ineficiencia no sea constante y decreciente (en el caso de la recaudación impositiva más seguridad social, si bien es positivo, no es significativo). Se analizan entonces los valores de lambda, que

13 No puede realizarse una comparación entre los modelos en sentido estricto porque estos corresponden a distintas especificaciones y no están anidados, es decir, no están incluidos cada uno en el anterior. 
Tabla 1 .

Determinantes de la recaudación potencial y explicación de la ineficiencia de los parámetros de la frontera estocástica tributaria por método de máxima verosimilitud

\begin{tabular}{|c|c|c|c|c|c|c|}
\hline Variables & $\begin{array}{c}\text { Battese y } \\
\text { Coelli (1992) } \\
\text { Halfnormal **** }\end{array}$ & $\begin{array}{l}\text { Battese y } \\
\text { Coelli (1995) } \\
\text { Truncated } \\
\text { normal }\end{array}$ & $\begin{array}{c}\text { Battese y } \\
\text { Coelli (1995) } \\
\text { Truncated } \\
\text { normal, } \\
\text { Heterogenous } \\
\text { in mean } \\
\text { and decay } \\
\text { inefficiency }\end{array}$ & $\begin{array}{c}\text { Battese y } \\
\text { Coelli }(1992) \\
\text { Halfnormal } * * * *\end{array}$ & $\begin{array}{l}\text { Battese y } \\
\text { Coelli (1995) } \\
\text { Truncated } \\
\text { normal }\end{array}$ & $\begin{array}{c}\text { Battese y } \\
\text { Coelli (1995) } \\
\text { Truncated } \\
\text { normal, } \\
\text { Heterogenous } \\
\text { in mean } \\
\text { and decay } \\
\text { inefficiency }\end{array}$ \\
\hline & 1 & 2 & 3 & 4 & 5 & 6 \\
\hline \multicolumn{7}{|c|}{ Estimación de frontera estocástica } \\
\hline \multirow[t]{2}{*}{ In_gdpc_ppp } & $-0,0583521$ & 0,1652932 & 0,0742412 & 0,0646165 & 0,1475009 & 0,2073218 \\
\hline & $(-1,464)$ & $(1,602)$ & $(0,907)$ & $(1,979)^{* *}$ & $(1,806)^{*}$ & $(1,741)^{*}$ \\
\hline \multirow[t]{2}{*}{ agr } & $-0,0023501$ & $-0,0136370$ & $-0,0168843$ & $-0,0010803$ & $-0,0154000$ & 0,0047993 \\
\hline & $(-0,864)$ & $(-1,116)$ & $(-1,708)^{*}$ & $(-0,375)$ & $(-1,524)$ & $(0,560)$ \\
\hline \multirow[t]{2}{*}{ trade } & 0,0000127 & $-0,0000481$ & & $-0,0004298$ & $-0,0011252$ & $-0,0019337$ \\
\hline & $(0,032)$ & $(-0,036)$ & & $(-0,789)$ & $(-1,456)$ & $(-2,704)^{* * *}$ \\
\hline \multirow[t]{2}{*}{ soc_edu } & & & & 0,0210236 & 0,1043717 & 0,1084729 \\
\hline & & & & $(2,951)^{* * *}$ & $(3,661)^{* * *}$ & $(5,017)^{* * *}$ \\
\hline \multirow[t]{2}{*}{ gini100 } & & & & $-0,0070954$ & & 0,0026267 \\
\hline & & & & $(-3,558)^{* * *}$ & & $(0,310)$ \\
\hline \multirow[t]{2}{*}{ dummy } & & 0,0373317 & & 0,0497919 & 0,0273911 & $-0,0346329$ \\
\hline & & $(0,594)$ & & $(1,618)$ & $(0,495)$ & $(-0,457)$ \\
\hline \multirow[t]{2}{*}{ Constant } & 4,0746095 & 1,9716865 & 2,9813278 & 3,1265038 & 1,5950830 & 0,8562371 \\
\hline & $(10,220)^{* * *}$ & $(1,855)^{*}$ & $(3,406)^{* * *}$ & $(8,423)^{* * *}$ & $(2,107)^{* *}$ & $(0,502)$ \\
\hline \multicolumn{7}{|c|}{ Estimación de ineficiencia } \\
\hline \multirow[t]{2}{*}{ complexity } & & & $-0,0047006$ & & & \\
\hline & & & $(-2,971)^{* * *}$ & & & \\
\hline \multirow[t]{2}{*}{ In_cpi } & & & & & & 0,0053601 \\
\hline & & & & & & $(0,199)$ \\
\hline \multirow[t]{2}{*}{ wgi_6 } & & & & & & $-0,2285098$ \\
\hline & & & & & & $(-1,498)$ \\
\hline \multirow[t]{2}{*}{ Constant } & & 0,1574642 & 0,7602840 & & $-6,3116380$ & 0,1028467 \\
\hline & & $(0,343)$ & $(5,756)^{* * *}$ & & $(-2,060)^{* *}$ & $(0,278)$ \\
\hline Observations & 551 & 551 & 551 & 409 & 465 & 255 \\
\hline Number of ncountry & 23 & 23 & 23 & 21 & 22 & 19 \\
\hline \multirow[t]{2}{*}{ sigma_u } & 0,399 & 0,321 & 0,221 & 0,356 & 0,866 & 0,118 \\
\hline & & $(2,69) * * *$ & $(6,07)^{* * *}$ & & $(3,26)^{* * *}$ & $(1,46)^{* *}$ \\
\hline II & 404,2 & $-21,13$ & 87,80 & 302,1 & 82,32 & 97,28 \\
\hline chi2 & 51,12 & 20,88 & 11,10 & 41,80 & 73,28 & 60,03 \\
\hline \multirow[t]{2}{*}{ sigma_v } & 0,104 & 0,129 & 0,0602 & 0,103 & 0,170 & 0,131 \\
\hline & & $(1,91)^{* *}$ & $(2,32)^{* * *}$ & & $(6,27)^{* * *}$ & $(2,22)^{* *}$ \\
\hline \multirow[t]{2}{*}{ lambda } & & 2,482831 & 3,675434 & & 5,083913 & 0,898734 \\
\hline & & $(18,15)^{* * *}$ & $(86,22)^{* * *}$ & & $(17,6)^{* * *}$ & $(6,58)^{* * *}$ \\
\hline \multirow[t]{2}{*}{ eta } & 0,0232402 & & & 0,0297648 & & \\
\hline & $(7,439)^{* * *}$ & & & $(8,258)^{* * *}$ & & \\
\hline
\end{tabular}

Nota: z-statistics entre paréntesis; ${ }^{* * *} p<0,01,{ }^{* *} p<0,05,{ }^{*} p<0,1 * * * *$ : dummy por periodo.

Fuente: cálculos del autor basados en datos de BID-CIAT, WDI y otros (ver tabla A.1). 
son significativos y reflejan la importancia de la ineficiencia en el término de error.

Al adicionar variables explicativas para el componente de ineficiencia, se encuentra que el parámetro de complejidad tributaria es significativo y tiene signo negativo; este se asocia negativamente con la ineficiencia y positivamente con la eficiencia. Los parámetros de inflación afectan positivamente la ineficiencia (efecto de rezago fiscal), mientras que el parámetro de corrupción tiene signo negativo. Como esta variable tiene valores crecientes a menor nivel de corrupción, una menor corrupción medida por este índice se asocia a una menor ineficiencia.

Davoodi y Grigorian (2007), por ejemplo, encuentran que la calidad institucional tiene un rol preponderante en la relación con la recaudación tributaria (captada aquí con el indicador de corrupción). En relación con la inflación, los resultados son también consistentes con Agbeyegbe, Stotsky y WoldeMariam (2004) y Davoodi y Grigorian (2007).

\section{LA PREDICCIÓN DEL ESFUERZO FISCAL DE LOS PAÍSES Y LOS NIVELES DE INEFICIENCIA}

En los apartados anteriores, este artículo se ha enfocado en intentar identificar factores que afectan la carga fiscal ajustada según la metodología BID-CIAT; el paso adicional es evaluar si los países estarían en condiciones de incrementar el nivel de recaudación actual o, por el contrario, si están recaudando muy cerca de su potencial.

Tabla 2.

Correlación entre las estimaciones de esfuerzo fiscal para la carga tributaria ajustada

\begin{tabular}{|c|c|c|c|c|c|c|}
\hline & Esfuerzo fiscal 1 & Esfuerzo fiscal 2 & Esfuerzo fiscal 3 & Esfuerzo fiscal 4 & Esfuerzo fiscal 5 & Esfuerzo fiscal 6 \\
\hline \multirow[b]{2}{*}{$\begin{array}{c}\text { Esfuerzo } \\
\text { fiscal } 1\end{array}$} & 1 & & & & & \\
\hline & & & & & & \\
\hline \multirow{2}{*}{$\begin{array}{c}\text { Esfuerzo } \\
\text { fiscal } 2\end{array}$} & 0,711 & 1 & & & & \\
\hline & 0,00 & & & & & \\
\hline \multirow{2}{*}{$\begin{array}{c}\text { Esfuerzo } \\
\text { fiscal } 3\end{array}$} & 0,7677 & 0,9659 & 1 & & & \\
\hline & 0,00 & 0,00 & & & & \\
\hline \multirow{2}{*}{$\begin{array}{c}\text { Esfuerzo } \\
\text { fiscal } 4\end{array}$} & 0,9598 & 0,8314 & 0,8558 & 1 & & \\
\hline & 0,00 & 0,00 & 0,00 & & & \\
\hline \multirow{2}{*}{$\begin{array}{l}\text { Esfuerzo } \\
\text { fiscal } 5\end{array}$} & 0,4891 & 0,7718 & 0,7038 & 0,5704 & 1 & \\
\hline & 0,00 & 0,00 & 0,00 & 0,00 & & \\
\hline \multirow{3}{*}{$\begin{array}{c}\text { Esfuerzo } \\
\text { fiscal } 6\end{array}$} & 0,7187 & 0,6039 & 0,6287 & 0,7285 & 0,7263 & 1 \\
\hline & 0,00 & 0,00 & 0,00 & 0,00 & 0,00 & \\
\hline & & & & & & \\
\hline
\end{tabular}

Nota: la significatividad de los coeficientes de correlación se muestra en letra cursiva.

Fuente: cálculos del autor en base a datos de BID-CIAT, WDI y otros. 
A partir de los coeficientes estimados en los modelos, se genera una recaudación fiscal predicha que será considerada como la recaudación potencial; la comparación entre la recaudación potencial y la efectiva determinará cuán cerca se encuentran en relación con su capacidad fiscal. Este enfoque tiene varias limitaciones (Davoodi y Grigorian, 2007), como, por ejemplo, factores inobservables que estén afectando la recaudación y no estén incluidos en las especificaciones de los modelos, la disponibilidad de información y que el nivel de recaudación potencial estimado varíe en función de la especificación elegida.

Utilizando las estimaciones de la tabla 1 , se predice el esfuerzo fiscal para cada país a través de la fórmula basada en Jondrow et al. (1982), dado el valor observable de Vit-Uit (Pessino y Fenochietto, $2010,2013)$ presentado en la ecuación [9]. Es interesante evaluar si las estimaciones difieren significativamente entre sí. En la tabla 2 puede apreciarse que tales estimaciones están correlacionadas en forma positiva (Karagiannis y Tzouvelekas, 2009) y son significativas en todos los casos.

Tabla 3

Estimación de la capacidad y el esfuerzo fiscal de los países de América Latina y el Caribe (promedios de las estimaciones en porcentaje del PIB)

\begin{tabular}{|c|c|c|c|c|}
\hline \multirow[t]{2}{*}{ País } & \multirow{2}{*}{$\begin{array}{c}\text { PIB } 2013 \text { per cápita PPP (U\$S } \\
\text { constantes 2005) }\end{array}$} & $\begin{array}{c}\text { Carga fiscal } \\
\text { ajustada }\end{array}$ & $\begin{array}{c}\text { Capacidad } \\
\text { fiscal promedio }\end{array}$ & Esfuerzo fiscal promedio \\
\hline & & \multicolumn{3}{|c|}{ Promedio 1990-2013 } \\
\hline Argentina & $22.362,83$ & 26,6 & 33,0 & 0,82 \\
\hline Bahamas & $24.648,34$ & 17,7 & 33,7 & 0,53 \\
\hline Barbados & $16.015,29$ & 31,1 & 35,2 & 0,90 \\
\hline Bélice & $8.013,55$ & 22,8 & 27,6 & 0,81 \\
\hline Bolivia & $5.927,93$ & 22,9 & 29,1 & 0,82 \\
\hline Brasil & $14.987,02$ & 30,8 & 33,4 & 0,93 \\
\hline Costa Rica & $13.427,26$ & 22,1 & 29,4 & 0,77 \\
\hline Chile & $22.533,54$ & 25,0 & 30,2 & 0,84 \\
\hline Colombia & $12.775,56$ & 19,2 & 27,5 & 0,72 \\
\hline República Dominicana & $12.173,06$ & 13,3 & 25,6 & 0,56 \\
\hline Ecuador & $10.908,02$ & 13,5 & 25,7 & 0,55 \\
\hline Guatemala & $7.289,73$ & 11,8 & 23,2 & 0,56 \\
\hline Honduras & $4.592,17$ & 18,2 & 25,5 & 0,73 \\
\hline Jamaica & $8.486,95$ & 23,2 & 29,6 & 0,80 \\
\hline México & $17.389,85$ & 20,3 & 29,3 & 0,71 \\
\hline Nicaragua & $4.432,33$ & 15,1 & 24,2 & 0,66 \\
\hline Panamá & $19.080,45$ & 19,6 & 27,9 & 0,71 \\
\hline Paraguay & $8.064,31$ & 18,2 & 25,9 & 0,73 \\
\hline Perú & $11.557,07$ & 18,2 & 27,0 & 0,70 \\
\hline Salvador & $7.782,53$ & 15,9 & 25,6 & 0,66 \\
\hline Trinidad y Tobago & $29.469,35$ & 27,0 & 33,7 & 0,81 \\
\hline Uruguay & $19.678,65$ & 28,5 & 31,5 & 0,90 \\
\hline Venezuela & $18.452,87$ & 20,3 & 31,7 & 0,66 \\
\hline Total & $13.915,16$ & 20,9 & 28,6 & 0,74 \\
\hline
\end{tabular}

Fuente: cálculos del autor basados en datos de BID-CIAT y CEPALSTAT. 
La tabla 3 presenta los resultados cuantitativos de las estimaciones, calculados a partir de promediar los resultados de cada una de las especificaciones presentadas. Se realizan las predicciones para cada una de las especificaciones que surgen de la tabla 1 y luego se promedian en la tabla 3, dado que las predicciones, como fue expuesto, están correlacionadas positiva y significativamente en todos los casos.

En esta tabla se expone, en la primera columna, la carga fiscal ajustada. En las columnas subsiguientes se presenta la capacidad fiscal, calculada mediante las predicciones de los modelos anteriores, y el esfuerzo fiscal, calculado como el cociente entre la recaudación efectiva ajustada en porcentaje del PIB y la capacidad fiscal [9].

En primer lugar, es importante destacar que, dado que las estimaciones difieren en función de la determinación de las especificaciones de los modelos, las formas funcionales y las variables explicativas, cada modelo arroja una estimación de esfuerzo fiscal y un índice de capacidad fiscal distinto. En segundo lugar, debe señalarse que los modelos no son estrictamente comparables y que las predicciones que resultan de estos no son determinísticas, sino que corresponden a un valor medio en el que debe considerarse el intervalo de confianza del error de la predicción. En tercer lugar, cabe apreciar que las estimaciones de esfuerzo fiscal resultan ligeramente mayores en los casos en los que se modela la ineficiencia en relación con determinadas variables explicativas en comparación con el inicial, donde esta decrece a tasa constante.

De la lectura de los resultados se advierte que existen países que a lo largo del periodo analizado muestran valores de esfuerzo fiscal muy elevados en relación con la capacidad fiscal, como es el caso de Brasil, Uruguay y Barbados. Este grupo podría completarse con países de esfuerzo fiscal también significativo, aunque ligeramente inferior que los casos anteriores, como Argentina, Chile y Bolivia.

En contraposición con los anteriores, existe un grupo de países cuyo aprovechamiento de la capacidad fiscal potencial es bajo en relación con el resto de países analizados, dado que aproximadamente, y para todo el periodo en estudio, han promediado un esfuerzo fiscal inferior al $60 \%$ de la capacidad fiscal; es el caso de países como Ecuador, Guatemala, República Dominicana y Bahamas.

Un análisis de los resultados explicitados determina que existan países con elevado nivel de esfuerzo fiscal y alto ingreso per cápita, y países con bajo aprovechamiento de la capacidad fiscal pero elevado nivel de ingreso. De acuerdo con lo esperado, países de mayor nivel de imposición y mayor ingreso per cápita presentan estimaciones de esfuerzo fiscal más elevados. Como se explica en Fenochietto y Pessino (2013), es posible que la demanda por gasto público sea aquí un determinante del elevado nivel de recaudación, lo que lleva a apelar a bases imponibles más amplias, tasas más elevadas o explotación de fuentes de recursos que incluyan a los recursos naturales.

Los países con esfuerzo fiscal bajo, como Bahamas, pero con un alto nivel de PIB per cápita constituyen una excepción que puede ser explicada también por una decisión de elección pública: tanto el impuesto al valor agregado como el impuesto a la renta de personas físicas no forman parte de los instrumentos de política durante el periodo analizado.

Por otro lado, países con un nivel de ingreso per cápita relativamente bajo en promedio para todo el periodo analizado pero con un nivel de imposición relativamente elevado, como Bolivia o Jamaica, o de ingresos medios con niveles de imposición elevados, como Brasil o Uruguay, se relacionan con tasas impositivas elevadas (para Brasil, en promedio, para todo el periodo, la alícuota del impuesto general sobre los consumos es del $20 \%$, mientras que para Uruguay es del $22 \%$ ), o con la presencia de ingresos provenientes de recursos naturales. La explicación también puede ser relacionada con una decisión de elección pública que indique un deseo del gobierno por ampliar la intervención del sector público en la economía.

Si bien en promedio se advierte que para América Latina y el Caribe los países han incrementado su esfuerzo fiscal, pueden apreciarse, como fue expresado en los párrafos anteriores, diferencias con respecto a cada caso particular. La presencia de ingresos provenientes de recursos 
naturales ha contribuido al incremento del esfuerzo fiscal por parte de los países de la región a partir de la última década. Este esfuerzo puede verse en el indicador de presión fiscal ajustada para los casos en que los recursos mencionados estén incluidos en los ingresos no tributarios (Bolivia, Trinidad y Tobago, Venezuela), mientras que en otros, estos ingresos están comprendidos en las bases imponibles de los impuestos tradicionales (Argentina).

Algunas explicaciones sobre el comportamiento del esfuerzo fiscal y su correlación con los cambios legislativos experimentados en los países de la región pueden verificarse a partir de la evaluación de los cambios normativos ocurridos en varios países (Rossignolo, 2016). En los últimos años se han verificado algunas reformas al impuesto sobre la renta, que han permitido incrementar el indicador de esfuerzo fiscal. En varios países (como Chile, Ecuador, El Salvador, el Estado Plurinacional de Bolivia, Honduras, México, Perú y Uruguay) hubo una tendencia a incrementar las tasas de los impuestos o a fijar tasas adicionales. En otros casos, las reformas procuraron ampliar la base imponible del impuesto sobre la renta; en particular se ha extendido la tributación al conjunto de ingresos del trabajo y de las rentas de capital, lo que en algunos casos incluye la aplicación de impuestos sobre los dividendos (Colombia, El Salvador, Guatemala, Jamaica, Nicaragua, Panamá, Perú y República Dominicana).

En el caso de Uruguay, por ejemplo, las rentas de trabajo se comenzaron a gravar por separado, con tasas progresivas del $10 \%$ al $30 \%$, y las rentas del capital, con una tasa proporcional del $12 \%$, excepto los dividendos que tributan a una tasa del $7 \%$. En Perú también se incorporaron algunos elementos de imposición dual, y desde 2009 las rentas del capital comenzaron a gravarse con una tasa proporcional del $6,25 \%$ y las rentas provenientes del trabajo comenzaron a ser gravadas con una escala progresiva de tasas (del 15\% al 30\%).

Entretanto, la República Dominicana y varios países de Centroamérica (El Salvador, Guatemala, Honduras, Nicaragua y Panamá) han fijado tasas que se sitúan entre un $10 \%$ y un $15 \%$ con el fin de gravar las rentas de capital, con tasas mayores para las utilidades empresariales, y tasas progresivas para las rentas del trabajo.

\section{CONCLUSIONES}

A partir del análisis de la evolución de la recaudación fiscal en América Latina, se observa que la región ha experimentado un comportamiento creciente en la última década; sin embargo, en promedio, la recaudación se encuentra aún lejos de los niveles alcanzados en los países desarrollados. En consecuencia, surge la pregunta de cómo incrementar la recaudación mediante la ampliación de las bases imponibles vigentes.

Una de las alternativas en análisis consiste en alcanzar lo que actualmente no está tributando: la estimación de la base imponible potencial de los impuestos debería incluir los gastos tributarios y las mediciones de evasión. Es muy complejo poder llevar a cabo mediciones con metodologías comparables a lo largo de los diferentes países, debido a las dificultades para delimitar las bases imponibles, su administración y las deficiencias en los sistemas de estadísticas.

Adicionalmente, con el objetivo de analizar la sustentabilidad de financiamiento de los gastos públicos, una herramienta de utilidad para los países resulta ser la evaluación sobre el potencial recaudatorio, dado que, si su recaudación actual está cercana a este, la expansión del gasto deberá ser financiada con otra fuente de ingresos no tributaria (deuda pública, entre otros).

La literatura define la capacidad fiscal de un país a la máxima recaudación alcanzable, en función de la capacidad extractiva de recursos por parte del sector público. Esta puede calcularse en términos relativos a las de otros países y en función de determinadas características. Consecuentemente, el esfuerzo fiscal refiere la capacidad de la hacienda para recaudar más impuestos conforme a esa capacidad. Este trabajo presenta una estimación mediante la metodología de frontera estocástica, 
con el objetivo de calcular el esfuerzo fiscal de los países de América Latina y el Caribe, a partir de la brecha entre la recaudación efectiva y la capacidad fiscal. La diferencia entre la recaudación potencial y la observada encierra tanto ineficiencias técnicas como variables de política discrecionales (tasas, bases imponibles, exenciones), pero también decisiones sobre el nivel de provisión de bienes públicos por parte de las comunidades y su financiamiento.

Como otras variables explicativas del esfuerzo fiscal fueron incorporadas, por un lado, el ingreso per cápita, la participación de la agricultura en el producto y la apertura comercial, mientras que, por otro lado, se agregaron a las variables anteriores el gasto en educación y la desigualdad medida a través del índice de Gini. Además, se incorporan como controles a variables de índole institucional, como la complejidad del sistema tributario y la inflación, un indicador para aproximar a la evolución de la corrupción, con el objetivo de modelar la ineficiencia.

Los resultados del estudio son consistentes con los antecedentes de la literatura en la temática; se corrobora la relación positiva y significativa entre la recaudación fiscal ajustada y el nivel de desarrollo (ingreso per cápita), apertura económica (importaciones y exportaciones como porcentaje del PIB) y educación (gasto público educativo como porcentaje del PIB).

La modelización del componente de ineficiencia presenta evidencia de que esta es decreciente (sin embargo, al considerar solo a los países con ingresos provenientes de recursos naturales, tal ineficiencia es creciente). La complejidad del sistema tributario se asocia negativamente con la ineficiencia, mientras que los parámetros de inflación afectan positivamente la ineficiencia y una menor corrupción medida se asocia a una menor ineficiencia.

La evidencia encontrada en este trabajo sugiere que existe una diferencia entre la recaudación efectiva y la potencial, que es mayor para los países de menor recaudación. Al comparar la recaudación predicha por los modelos con la efectiva, emergen heterogeneidades entre los distintos países.

Existen casos de países con elevada presión fiscal, como Brasil, que arroja valores muy cercanos al nivel de recaudación potencial. En otros casos (Paraguay, Uruguay, Perú), la incorporación reciente del impuesto a la renta de personas físicas los aproxima en mayor medida al potencial recaudatorio máximo y muestra claramente el esfuerzo de incrementar los ingresos fiscales por parte de estos países. Sin embargo, los países con ingresos provenientes de recursos naturales dejan más expuesta la generación de recursos a las oscilaciones de los precios internacionales, lo que afecta en una magnitud considerable el esfuerzo fiscal.

En consecuencia, la determinación sobre la capacidad fiscal depende de las particularidades de cada economía; se evidencia que los países con menor nivel de desarrollo están asociados a una menor capacidad de generación de recursos fiscales, y que otros países con desarrollo medio o alto podrían incrementar su recaudación gravando en una mayor medida sus actividades económicas, pero por decisiones políticas e institucionales las sociedades han decidido no hacerlo o mantener tasas impositivas bajas.

\section{REFERENCIAS}

1. Aigner, D., Lovell, C. y Schmidt, P. (1977). Formulation and estimation of stochastic frontier production function models. Journal of Econometrics, 6(1), 21-37.

2. Agbeyegbe, T., Stotsky, J. y WoldeMariam, A. (2004). Trade liberalization, exchange rate changes, and tax revenue in Sub-Saharan Africa. IMF Working Paper 04/178.

3. Alfirman, L. (2003). Estimating stochastic frontier tax potential: Can Indonesian Local Governments increase tax revenues under decentralization? IMF Working Paper 3/19. 
4. Alm. J. y Duncan, D. (2013). Estimating tax agency efficiency. Public Budgeting Ë Finance, 34(3), 92-110.

5. Aronson, R. y Hilley, J. (1986). Financing state and local governments (4. ${ }^{\mathrm{a}}$ ed.). Washington: The Brookings Institution.

6. Artana, D. y Templado, I. (2010). Is Argentina tax revenue effort too high? (Documento de Trabajo 106). Buenos Aires: Fundación de Investigaciones Económicas Latinoamericanas.

7. Barreix, A., Benítez, J., Velayos, F., Bes, M. y Pecho, M. (2013). Metiendo presión: estimando la verdadera carga fiscal de América Latina y el Caribe. La presión fiscal equivalente (Papeles de Trabajo 9). Washington: Banco Interamericano de Desarrollo e Instituto de Estudios Fiscales.

8. Barreix, A., Bes, M. y Roca, J. (2009). Equidad fiscal en Centroamérica, Panamá y República Dominicana. Washington: Banco Interamericano de Desarrollo y Eurosocial.

9. Barreix, A., Roca, J. y Villela, L. (2006). La equidad fiscal en los países andinos. Washington: Banco Interamericano de Desarrollo.

10. Battese, G. y Coelli, T. (1988). Prediction of firm level technical efficiencies with generalized frontier production function and panel data. Journal of Econometrics, 38(3), 387-399.

11. Battese, G. y Coelli, T. (1992). Frontier production functions, technical efficiency and panel data: With application to paddy farmers in India. The Journal of Productivity Analysis, 3, 153-169

12. Battese, G. y Coelli, T. (1995). A model for technical inefficiency effects in a stochastic frontier production function for panel data. Empirical Economics, 20(2), 325-332.

13. Battese, G. y Corra, G. (1977). Estimation of a production frontier model: With application to the pastoral zone of Eastern Australia. The Australian Journal of Agricultural and Resource Economics, 21 (3), 169-179.

14. Bird, R., Martínez-Vázquez, J. y Torgler B. (2004). Societal institutions and tax effort in developing countries. En J. Alm y J. Martínez-Vázquez (Eds.), The challenge of tax reform in the global economy. Springer-Verlag.

15. Bird, R., Martínez-Vázquez, J. y Torgler, B. (2008). Tax effort in developing countries and high income countries: the impact of corruption, voice and accountability. Economic Analysis and Policy, 38(1).

16. Comisión Económica para América Latina y el Caribe (Cepal) (2006). La protección social de cara al futuro: acceso, financiamiento y solidaridad. Montevideo: Cepal.

17. Comisión Económica para América Latina y el Caribe (Cepal) (2013). Recursos naturales: situación y tendencias para una agenda de desarrollo regional en América Latina y el Caribe. Santiago de Chile: Autor.

18. Comisión Económica para América Latina y el Caribe (Cepal) (2014a). Balance preliminar de las economías de América Latina y el Caribe. Santiago de Chile: Autor.

19. Comisión Económica para América Latina y el Caribe (Cepal) (2014b). Pactos para la igualdad. Hacia un futuro sostenible. Lima: Autor.

20. Cetrángolo, O. y Gómez Sabaini, J. (2009). La imposición en Argentina: un análisis de la imposición a la renta, los patrimonios y otros tributos considerados directos. Macroeconomía del Desarrollo, 84

21. Cetrángolo, O., Gómez Sabaini, J. y Morán, D. (2014). La evasión contributiva en la protección social de salud y pensiones. Un análisis para Argentina, Colombia y Perú. CEPAL, Serie Políticas Sociales, 208

22. Cetrángolo, O., Gómez Sabaini, J. y Morán, D. (2015). Argentina: reformas fiscales, crecimiento e inversión (2000-2014). Macroeconomía del Desarrollo, 165. 
23. Coelli, T. (1996). A guide to FRONTIER version 4.1: a computer program for stochastic frontier production and cost function estimation. CEPA Working Paper, 96(7).

24. Cyan, M., Martínez-Vázquez, J. y Vulovic, V. (2013). Measuring tax effort: Does the estimation approach matter and should effort be linked to expenditureg oals? International Center for Public Policy Working Paper, 13 (8).

25. Davoodi, H. y Grigorian, G. (2007). Tax potential vs. tax effort: a cross-country analysis of Armenia's stubbornly low tax collection. IMF Working Paper 7/106.

26. Di Gresia, L. (2004). Desempeño comparado impuesto al valor agregado e impuesto sobre los ingresos brutos. En A. Porto (Ed.), Disparidades regionales y federalismo fiscal (pp. 325-335). La Plata: Universidad de La Plata

27. Esteller-Moré, A. (2005). Is there a connection between the tax administration and the politica power? International Tax and Public Finance, 12(5).

28. Filippini, M. y Greene, W. (2014). Persistent and transient productive inefficiency: a maximum simulated likelihood approach. Economics Working Paper, 14(197).

29. Garg, S., Goyal, A. y Pal, R. (2014). Why tax effort falls short of capacity in Indian states: A stochastic frontier approach. Working Paper2014-032. Mumbay: Indira Gandhi Institute of Development Research.

30. Gómez Sabaini, J. y Rossignolo, D. (2014). La tributación sobre las altas rentas en América Latina. Estudios y Perspectivas, 13.

31. Gómez Sabaini, J., Harriague, M. y Rossignolo, D. (2013). La situación fiscal en Argentina y sus efectos en la distribución del ingreso. Una estimación para el año 2008. Desarrollo Económico, 52 (207-208),341-382.

32. Goñi, E., López, H. y Servén, L. (2008). Fiscal redistribution and income inequality in Latin America. World Development, 39(9), 1558-1569.

33. Gordon, R. y Li, W. (2009). Tax structures in developing countries; many puzzles and a possible explanation. Journal of Public Economics, 93(7-8), 855-866.

34. Gupta, A. (2007). Determinants of tax revenue efforts in developing countries (IMF Working Paper 7/184). Washington: Fondo Monetario Internacional.

35. Fondo Monetario Internacional (FMI) (2014). Fiscal policy and income inequality. Recuperado de https:// www.imf.org/external/np/pp/eng/2014/012314.pdf

36. Jaimovich, D. y Panizza, U. (2007). Procyclicality or reverse causality? (Working Paper 599). Washington: Banco Interamericano de Desarrollo.

37. Jiménez, J., Gómez, J. y Podestá, A. (2010). Tributación, evasión y equidad en América Latina y el Caribe. En J. Jiménez, y Gómez Sabainiy A. Podestá (Eds.). Evasión y equidad en América Latina. Santiago de Chile: Cepal.

38. Jiménez, J., Gómez Sabaini, J. y Rossignolo, D. (2012). Imposición a la renta personal y equidad en América Latina. Nuevos desafíos. Macroeconomía del Desarrollo, 119.

39. Jondrow, J., Lovell, C., Materov, I. y Schmidt, P. (1982). On the estimation of technical inefficiency in the stochastic frontier production function model. Journal of Econometrics, 19(2-3), 233-238.

40. Karagiannis, G. y Tzouvelekas, V. (2009). Parametric measurement of time-varying technical inefficiency: results from competing models. Agricultural Economics Review, 10(1), 50-79. 
41. Kenney, L. y Winner, S. (2006). Tax systems in the world: An empirical investigation into the importance of tax bases, collection costs, and political regime. International Tax and Public Finance, 13 (2-3), 181-215.

42. Kumbhakar, S., Lien, G. y Hardaker, J. (2014). Technical efficiency in competing panel data models: a study of Norwegian grain farming. Journal of Productivity Analysis, 41 (2), 321-337.

43. Langford, B. y Ohlenburg, T. (2015). Tax revenue potential and effort. An empirical investigation. International Growth Centre Working Paper.

44. Le, T., Moreno-Dodson, B. y Bayraktarc, N. (2012). Tax capacity and tax effort: Extended cross-country analysis from 1994 to 2009. Policy Research Working Paper, 6252.

45. Le, T., Moreno-Dodson, B. y Rojchaichaninthorn, J. (2008). Expanding taxable capacity and reaching revenue potential: cross-country analysis. Policy Research Working Paper, 4559.

46. López-Calva, L. y Lustig, N. (2010). Declining Latin American inequality: market forces or state action? Recuperado de: http://voxeu.org/article/declining-latin-american-inequality-market-forces-or-state-action

47. Mastromarco, C. (2008). Stochastic Frontier Models. Italia: University of Salento.

48. Organización para la Cooperación y el Desarrollo Económicos (OCDE) (2015). OECD Economic Surveys. Colombia: Autor.

49. Pecho, M., Peláez, F. y Sánchez, J. (2012). Estimación del incumplimiento tributario en América Latina 2000-2012 (Documento de trabajo 3). CIAT

50. Perry, G., Arias, O., Lopez, H., Maloney, W. y Serven, L. (2006). Poverty reduction and growth: virtuous and vicious circles. Washington: Banco Mundial.

51. Pessino, C. y Fenocchietto, R. (2010). Determining Countries' tax effort. Revista de Economía Pública, 195(4), 65-87.

52. Pessino, C. y Fenocchietto, R. (2013). Understanding countries' tax effort. International Monetary Fund Working Paper, 13/244.

53. Piancastelli, M. (2001). Measuring the tax effort of developedand developing countries. Cross country panel data analysis — 1985/95. Texto para Discussão, 818.

54. Rossignolo, D. (2012). Estimación de la recaudación potencial del impuesto a la renta en América Latina. Macroeconomía del Desarrollo, 120.

55. Rossignolo, D. (2015). Efectos económicos y macrofiscales de los recursos naturales en América Latina. Macroeconomía del Desarrollo, 170.

56. Rossignolo, D (2016). El impuesto a larRenta personal y los altos ingresos en América Latina. Review of Public Economics, 214(3), 115-148.

57. Rossignolo, D. y Gómez Sabaini, J. (2011). Impacto de las políticas tributarias sobre la equidad en países de América Latina. En J. Nun (Ed.), La desigualdad y los impuestos: introducción para no especialistas (II). Buenos Aires: Capital Intelectual.

58. Stein, E., Talvi, E. y Grisanti, A. (1998). Institutional arrangements and fiscal performance: The Latin American experience. IDB Working Paper, 367.

59. Stevenson, R. (1980). Likelihood functions for generalizad stochastic frontier estimation. Journal of Econometrics, 13(1), 57-66 
60. Stotsky, J. y WoldeMariam, A. (1997). Tax effort in the Sub-Saharan Africa. International Monetary Fund, Working Paper, 97/107.

61. Stotsky, J. y WoldeMariam, A. (2002). Central American tax reform: Trends and Possibilities. International Monetary Fund Working Paper, 2/227.

62. Teera, J. (2001). Tax performance: a comparative study. Bath: Universidad de Bath.

63. Villela L., Lemgruber, A. y Jorrat, M. (2010). Tax expenditure budgets. Concepts and challenges for implementation. IDB Working Paper, 179.

64. Wooldridge, J. (2002). Econometric analysis of cross section and panel data. Cambridge: MIT Press. 


\section{ANEXOS}

\section{Anexo 1}

Fuentes de información de las variables empleadas en el análisis

\begin{tabular}{|c|c|}
\hline Variable & Descripción y fuente \\
\hline $\begin{array}{l}\text { adjusted_tax } \\
\text { burden }\end{array}$ & $\begin{array}{l}\text { Carga fiscal ajustada como porcentaje del PIB. } \\
\text { Fuente: BID-CIAT. }\end{array}$ \\
\hline gdpc_ppp & $\begin{array}{c}\text { Producto Interno Bruto per cápita en paridad de poder de compra PPP } \\
\text { (dólares internacionales constantes 2005). } \\
\text { Fuente: World Development Indicators (WDI). }\end{array}$ \\
\hline agr & $\begin{array}{l}\text { Valor agregado por la agricultura (en porcentaje del PIB). } \\
\text { Fuente: WDI. }\end{array}$ \\
\hline trade & $\begin{array}{l}\text { Importaciones más exportaciones de bienes y servicios (\% del PIB). } \\
\text { Fuente: WDI }\end{array}$ \\
\hline complexity & 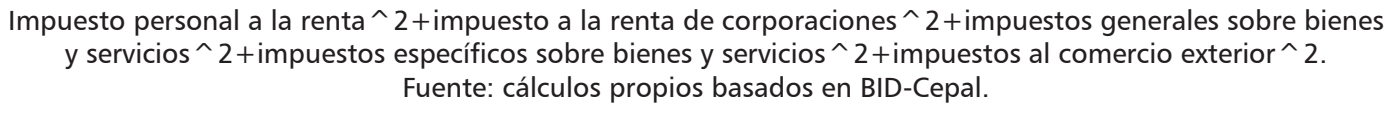 \\
\hline pop_growth & $\begin{array}{l}\text { Crecimiento poblacional (\% anual). } \\
\text { Fuente: WDI. }\end{array}$ \\
\hline soc_edu & $\begin{array}{l}\text { Gasto público en educación (\% del PIB). } \\
\text { Fuente: CEPALSTAT, WDI y Unesco. }\end{array}$ \\
\hline gini & $\begin{array}{l}\text { Índice de Gini de desigualdad en la distribución del ingreso. } \\
\text { Fuente: SEDLAC, WIID, CEPALSTAT, WDI e IDLA; algunos datos faltantes fueron obtenidos por interpolación. }\end{array}$ \\
\hline срі & $\begin{array}{l}\text { Inflación medida por el índice de precios al consumidor promedio. Los datos de inflación son promedios } \\
\text { anuales. El índice está basado en porcentajes anuales de crecimiento. } \\
\text { Fuente: World Economic Outlook, WDI. }\end{array}$ \\
\hline wgi_6 & $\begin{array}{l}\text { Control de corrupción varía entre }-2,5 \text { y 2,5. } \\
\text { Fuente: Worldwide Governance Indicators. }\end{array}$ \\
\hline
\end{tabular}




\section{Anexo 2}

Estadísticas descriptivas

\begin{tabular}{|c|c|c|c|c|c|}
\hline Variable & Obs & Media & Desvío Std. & Min & 0 \\
\hline adjusted_tax_burden & 552 & 20,926 & 6,182942 & 36,0928 \\
\hline gdpc_ppp & 552 & 9350,03 & 5544,073 & 2071,541 & 30874,91 \\
\hline agr & 552 & 9,88186 & 6,016421 & 0,3784971 & 25,87916 \\
\hline trade & 552 & 71,61361 & 33,27991 & 13,75305 & 198,7668 \\
\hline complexity & 552 & 65,24312 & 44,79053 & 0 & 296,9733 \\
\hline pop_growth & 552 & 1,509553 & 0,6879821 & $-0,0627996$ & 2,975644 \\
\hline soc_edu & 466 & 4,012923 & 1,395726 & 0,83 & 8,01 \\
\hline gini & 442 & 51,4267 & 4,866272 & 38,27 & 67,59 \\
\hline cpi & 517 & 44,9171 & 377,2112 & $-3,7$ & 7481,664 \\
\hline wgi_6 & 345 & $-0,142860$ & 0,7696263 & $-1,445456$ & 1,764707 \\
\hline
\end{tabular}

